Article

\title{
A Set Based Newton Method for the Averaged Hausdorff Distance for Multi-Objective Reference Set Problems
}

\author{
Lourdes Uribe $^{1, *} \mathbb{C}$, Johan M Bogoya ${ }^{2}{ }^{\oplus}$, Andrés Vargas ${ }^{2} \oplus$, Adriana Lara ${ }^{1} \oplus$, Günter Rudolph ${ }^{3}$ \\ and Oliver Schütze ${ }^{4}$ \\ 1 Instituto Politécnico Nacional, Mexico City 07738, Mexico; yadriana@esfm.ipn.mx \\ 2 Departamento de Matemáticas, Pontificia Universidad Javeriana, Cra. 7 N. 40-62, \\ Bogotá D.C. 111321, Colombia; jbogoya@javeriana.edu.co (J.M.B.); a.vargasd@javeriana.edu.co (A.V.) \\ 3 Department of Computer Science, TU Dortmund University, 44227 Dortmund, Germany; \\ Guenter.Rudolph@tu-dortmund.de \\ 4 Department of Computer Science, Cinvestav-IPN, Mexico City 07360, Mexico; schuetze@cs.cinvestav.mx \\ * Correspondence: lourdesur@esfm.ipn.mx
}

Received: 4 September 2020; Accepted: 11 October 2020; Published: 17 October 2020

check for updates

\begin{abstract}
Multi-objective optimization problems (MOPs) naturally arise in many applications. Since for such problems one can expect an entire set of optimal solutions, a common task in set based multi-objective optimization is to compute $N$ solutions along the Pareto set/front of a given MOP. In this work, we propose and discuss the set based Newton methods for the performance indicators Generational Distance (GD), Inverted Generational Distance (IGD), and the averaged Hausdorff distance $\Delta_{p}$ for reference set problems for unconstrained MOPs. The methods hence directly utilize the set based scalarization problems that are induced by these indicators and manipulate all $N$ candidate solutions in each iteration. We demonstrate the applicability of the methods on several benchmark problems, and also show how the reference set approach can be used in a bootstrap manner to compute Pareto front approximations in certain cases.
\end{abstract}

Keywords: multi-objective optimization; Newton method; performance indicator $\Delta_{p}$; generational distance; inverted generational distance; set based optimization

\section{Introduction}

Multi-objective optimization problems (MOPs), i.e., problems where multiple incommensurable and conflicting objectives have to be optimized concurrently, arise in many fields such as engineering and finance (e.g., [1-5]). One important characteristic is that there is typically not one single solution to be expected for such problems (as it is the case for "classical" scalar optimization problems (SOPs)), but rather an entire set of solutions. More precisely, if the MOP contains $k$ conflicting objectives, one can expect the solution set (the Pareto set respectively its image, the Pareto front) to form at least locally a manifold of dimension $k-1$ [6]. Many numerical methods take this fact into account and generate an entire (finite) set of candidate solutions so that the decision maker (DM) obtains an overview of the possible realizations of his/her project. For such set based multi-objective optimization algorithms a natural question that arises is the goodness of the obtained solution set $A$ (i.e., the relation of $A$ to the Pareto set/front of the underlying MOP). For this, several performance indicators have been proposed over the last decades such as the Hypervolume indicator (HV, [7]), the Generational Distance (GD, [8]), the Inverted Generational Distance (IGD, [9]), R2 [10], DOA [11], and the averaged Hausdorff distance $\Delta_{p}[12,13]$. Each such indicator assigns to a given set of candidate solutions an indicator value 
according to the given MOP. Hence, if the MOP and the size of the candidate solution set are fixed, the detection of the "best" candidate solution can be expressed by the problem

$$
\min _{\substack{A \subset Q \\|A|=N}} I(A),
$$

where $I$ denotes the chosen performance indicator (to be minimized), $Q \subset \mathbb{R}^{n}$ the domain of the objective functions, and $N$ the size of the candidate solution set. Since $A \subset \mathbb{R}^{n}$ contains $N$ elements, it is also a vector in $\mathbb{R}^{N \cdot n}$. Problem (1) can hence be regarded as a SOP with $N \cdot n$ decision variables.

A popular and actively researched class of set based multi-objective algorithms is given by specialized evolutionary algorithms, called multi-objective evolutionary algorithms (MOEAs, e.g., [14-17]). MOEAs evolve entire sets of candidate solutions (called populations or archives) and are hence capable of computing finite size approximations of the entire Pareto set/front in one single run of the algorithm. Further, they are of global nature, very robust, and require only minimal assumptions on the model (e.g., no differentiability on the objective or constraint functions). MOEAs have caught the interest of many reseachers and practitioners during the last decades, and have been applied to solve many real-world problems coming from science and engineering. It is also known, however, that none of the existing MOEAs converges in the mathematical sence which indicates that they are not yet tapping their full potential. In [18], it has been shown that for any strategy where $\lambda<\mu$ children are chosen from $\mu$ parents, there is no guarantee for convergence w.r.t. the HV indicator. Studies coming from mathematical programming (MP) indicate similar results for any performance indicator (e.g., $[19,20]$ ) since $\lambda<\mu$ strategies in evolutionary algorithms are equivalent to what is called cyclic search in MP.

In this work, we propose the set based Newton method for Problem (1), where we will address the averaged Hausdorff distance $\Delta_{p}$ as indicator. Since $\Delta_{p}$ is defined via GD and IGD, we will also consider the respective set based GD and IGD Newton methods. To this end, we will first derive the (set based) gradients and Hessians for all indicators, and based on this define and discuss the resulting set based Newton methods for unconstrained MOPs. Numerical results on some benchmark test problems indicate that the method indeed yields local quadratic convergence on the entire set of candidate solutions in certain cases. The Newton methods are tested on aspiration set problems (i.e., the problem to minimize the distance of a set of solutions toward a given utopian reference set $\mathrm{Z}$ and the given unconstrained MOP). Further, we will show how the $\Delta_{p}$ Newton method can be used in a bootstrap manner to compute finite size approximations of the entire Pareto front of a given problem in certain cases. The method can hence in principle be used as standalone algorithm for the treatment of unconstrained MOPs. On the other hand, the results also show that the Newton methods - as all Newton variants-are of local nature and require good initial solutions. In order to obtain a fast and reliable solver a hybridization with a global strategy-e.g., with MOEAs since the proposed Newton methods can be viewed as particular " $\lambda=\mu$ " strategies—seems to be most promising which is, however, beyond the scope of this work.

The remainder of this work is organized as follows: In Section 2, we will briefly present the required background needed for the understanding of this work. In Sections 3-5, we will present and discuss the set based GD, IGD and $\Delta_{p}$ Newton methods, respectively. Finally, we will draw our conclusions and will give possible paths for future work in Section 6.

\section{Background and Related Work}

Continuous unconstrained multi-objective optimization problems are expressed as

$$
\min _{x} F(x),
$$

where $F: \mathbb{R}^{n} \rightarrow \mathbb{R}^{k}, F(x)=\left(f_{1}(x), \ldots, f_{k}(x)\right)^{T}$ denotes the map that is composed of the individual objectives $f_{i}: \mathbb{R}^{n} \rightarrow \mathbb{R}, i=1, \ldots, k$, which are to be minimized simultaneously. 
If $k=2$ objectives are considered, the resulting problem is termed a bi-objective optimization problem (BOP).

For the definition of optimality in multi-objective optimization, the notion of dominance is widely used: for two vectors $a, b \in \mathbb{R}^{k}$ we say that $a$ is less than $b$ (in short: $a<_{p} b$ ), if $a_{i}<b_{i}$ for all $i \in\{1, \ldots, k\}$. The definition of $\leq_{p}$ is analog. Let $x, y \in \mathbb{R}^{n}$, then we say that $x$ dominates $y(x \prec y)$ w.r.t (2) if $F(x) \leq_{p} F(y)$ and $F(x) \neq F(y)$. Else, we say that $y$ is non-dominated by $x$. Now we are in the position to define optimality of a MOP. A point $x^{*} \in \mathbb{R}^{n}$ is called Pareto optimal (or simply optimal) w.r.t. (2) if there exists no $y \in \mathbb{R}^{n}$ that dominates $x^{*}$. We denote by $P$ the set of all optimal solutions, also called Pareto set. Its image $F(P)$ is called the Pareto front. Under mild conditions on the MOP one can expect that both sets form at least locally objects of dimension $k-1$ [6].

The averaged Hausdorff distance $\Delta_{p}$ for discrete or discretized sets is defined as follows: let $A=\left\{a_{1}, \ldots, a_{N}\right\}$ and $B=\left\{b_{1}, \ldots, b_{M}\right\}$, where $A, B \subset \mathbb{R}^{n}$, be finite sets. The values $G D_{p}(A, B)$ and $\operatorname{IGD}(A, B)$ are defined as

$$
\begin{aligned}
G D_{p}(A, B) & :=\left(\frac{1}{N} \sum_{i=1}^{N} \operatorname{dist}\left(a_{i}, B\right)^{p}\right)^{1 / p} \\
\operatorname{IGD}_{p}(A, B) & :=\left(\frac{1}{M} \sum_{i=1}^{M} \operatorname{dist}\left(b_{i}, A\right)^{p}\right)^{1 / p},
\end{aligned}
$$

where $p$ is an integer and where the distance of a point $a_{i}$ to a set $B$ is defined by $\operatorname{dist}\left(a_{i}, B\right):=$ $\min _{b \in B}\left\|a_{i}-b\right\|_{2}$. The averaged Hausdorff distance $\Delta_{p}$ is simply the maximum of these two values,

$$
\Delta_{p}:=\max \left\{G D_{p}(A, B), I G D_{p}(A, B)\right\} .
$$

We refer to [21] for an extension of the indicators to continuous sets. We stress that all of these three indicators are entirely distance based and are in particularly not Pareto compliant. A variant of IGD that is weakly Pareto compliant is the indicator DOA. Here, we are particularly interested in multi-objective reference set problems. That is, given a finite reference set $Z \subset \mathbb{R}^{k}$, we are interested in solving the problem

$$
\min _{\substack{A \subset Q \\|A|=N}} I(F(A), Z),
$$

where I is one of the indicators $G D_{p}, I G D_{p}$, or $\Delta_{p}$, and $N$ is the size of the approximation.

Probably the most important reference set in our context is the Pareto front itself. For this case, $\Delta_{p}$ prefers, roughly speaking, evenly spread solutions along the Pareto front and is hence e.g., in accord with the terms spread and convergence as used in the evolutionary multi-objective optimization (EMO) community for a "suitable" performance indicator. As an example, Figure 1 shows some "best approximations" in the $\Delta_{2}$ sense (i.e., when using $p=2$ ) for MOPs with different shapes of the Pareto front. More precisely, each subfigure shows a fine grain $(M=200)$ approximation of the Pareto front of the underlying problem (using dots), as well as the best approximations in the $\Delta_{2}$ sense (using diamonds). The latter are (numerical) solutions of (5) for $N=20$, and where $Z$ has been chosen as the Pareto front approximation. 

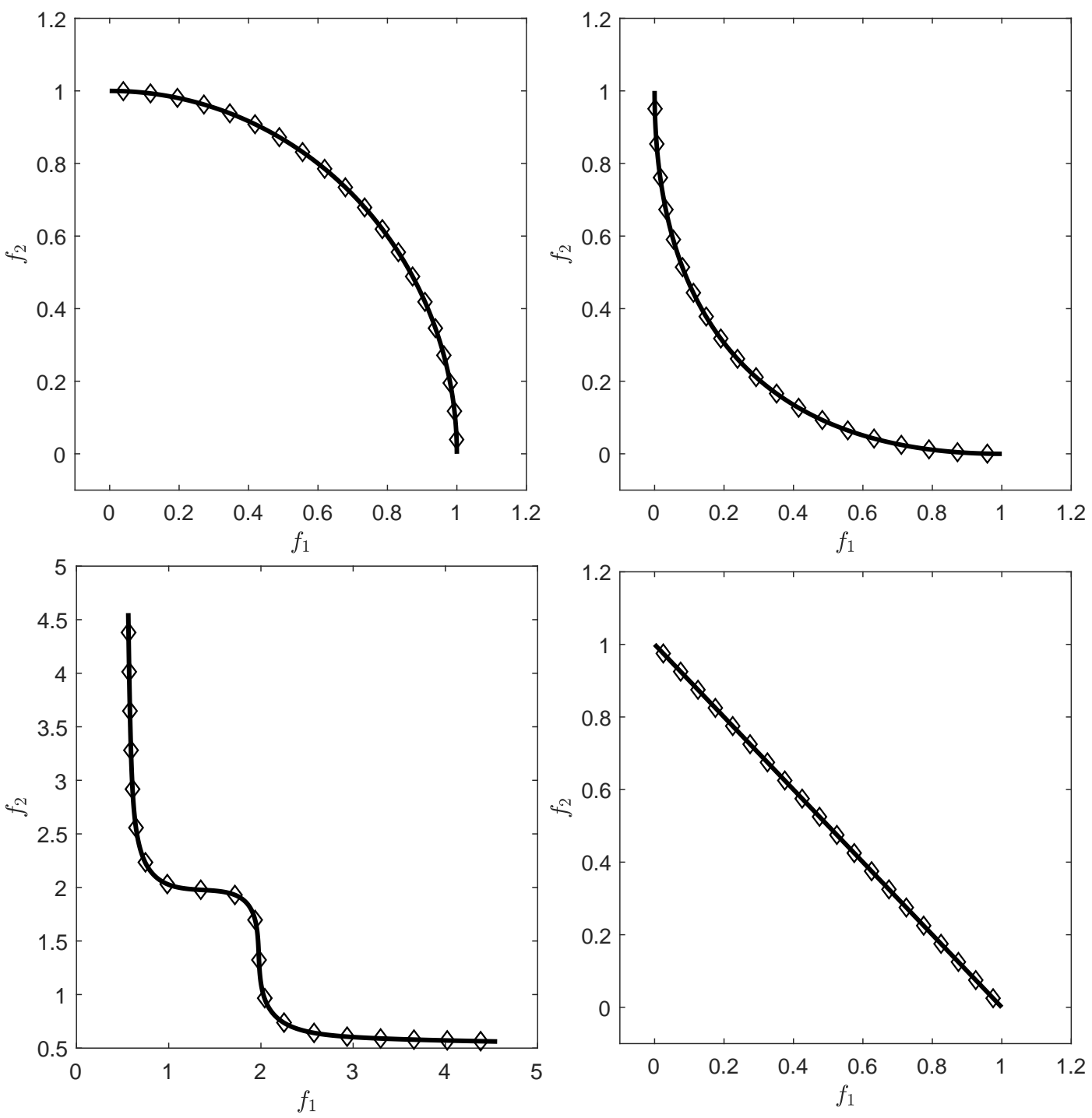

Figure 1. Pareto fronts with different shapes together with their best approximations in the sense of (5) for $p=2$ and $N=20$, and where $Z$ is an approximation of the Pareto front.

If $A=\left\{a_{1}, \ldots, a_{N}\right\}$ is a subset of the $\mathbb{R}^{n}$ it means that each of its element $a_{i}$ is an element of the $\mathbb{R}^{n}$. Hence, the set $A=\left\{a_{1}, \ldots, a_{N}\right\} \subset \mathbb{R}^{n}$ can in a natural way also be identified as a point or vector in the higher dimensional space $\mathbb{R}^{N \cdot n}$, i.e., $A \in \mathbb{R}^{N \cdot n}$. That is, the optimization problem (5) can be identified as a "classical" scalar optimization problem that is defined in $N \cdot n$-dimensional search space. A necessary condition for optimality is hence given by the Karush-Kuhn-Tucker conditions, e.g., for unconstrained problems we are seeking for sets $A$ for those the (set based) gradient vanishes. In order to solve this root finding problem, one can e.g., utilize the Newton method. If we are given a performance indicator $I$ together with the derivatives $\nabla I(A)$ and $\nabla^{2} I(A)$ on a set $A$, the Newton function is hence given by

$$
N(A):=A-\nabla^{2} I(A)^{-1} \nabla I(A) .
$$

There exist many methods for the computation of Pareto optimal solutions. For example, there are mathematical programming (MP) techniques such as scalarization methods that transform the MOP into a sequence of scalar optimization problems (SOPs) [22-26]. These methods are very efficient in finding a single solution or even a finite size discretization of the solution set. Another sub-class of the MP techniques is given by continuation-like methods that take advantage of the fact that the Pareto set forms - at least locally-a manifold. Methods of this kind start from a given initial solution and perform a search along the solution manifold [6,27-33]. 
Next there exist also set oriented methods that are capable of obtaining the entire solution set in a global manner. Examples for the latter are subdivision [34-36] and cell mapping techniques [37-39]. Another class of set based methods is given by multi-objective evolutionary algorithms (MOEAs) that have proven to be very effective for the treatment of MOPs [14,16,40-43]. Some reasons for this include that are very robust, do not require hard assumptions on the model, and allow to compute a reasonable finite size representation of the solution set already in a single run.

Methods that deal with single reference points for multi-objective problems can be found in $[26,44,45]$. The first work that deals with a set based approach using a problem similar to the one in (5) can be found in [46], where the authors apply the steepest descent method on the Hypervolume indicator [47]. In [48], the Newton method is defined where as well the Hypervolume indicator has been used. In [49], a multi-objective Newton method is proposed that detects single Pareto optimal solutions for a given MOP. In [50], a set based Newton method is proposed for general root finding problems and for convex sets.

\section{3. $G D_{p}$ Newton Method}

In the following sections we will investigate the set based Newton methods for $G D_{p}, I G D_{p}$, and $\Delta_{p}$. More precisely, we will consider the $p$-th powers, $p>1$, of these indicators as this does not change the optimal solutions. In all cases, we will first derive the (set based) derivatives, and then investigate the resulting Newton method. For the derivatives, we will focus on $p=2$ which is related to the Euclidean norm, and which hence represents the most important performance indicator of the indicator families. However, we will also state the derivatives for general integers $p$.

Let $A=\left\{a_{1}, \ldots, a_{N}\right\} \subset \mathbb{R}^{n}$ be a candidate set for (2), and $Z=\left\{z_{1}, \ldots, z_{M}\right\} \subset \mathbb{R}^{k}$ be a given reference set. The indicator $G D_{p}$ measures the averaged distance of the image of $A$ and $Z$ :

$$
G D_{p}(A):=\left(\frac{1}{N} \sum_{i=1}^{N} d\left(F\left(a_{i}\right), Z\right)^{p}\right)^{\frac{1}{p}} .
$$

Hereby, we have used the notation

$$
d\left(F\left(a_{i}\right), Z\right):=\min _{j=1, \ldots, M}\left\|F\left(a_{i}\right)-z_{j}\right\|, \text { for } i=1, \ldots, N,
$$

and assume $Z$ to be fixed for the given problem (and hence, it does not appear as input argument).

\subsection{Derivatives of $G D_{2}^{2}$}

\subsubsection{Gradient of $G D_{2}^{2}$}

In the following, we have to assume that for every point $F\left(a_{i}\right)$ there exists exactly one closest element in $Z$. That is, $\forall i=1, \ldots, N$ there exists an index $j_{i} \in\{1, \ldots, M\}$ such that:

$$
d\left(F\left(a_{i}\right), Z\right)=\left\|F\left(a_{i}\right)-z_{j_{i}}\right\|<\left\|F\left(a_{i}\right)-z_{q}\right\| \forall q \in\{1, \ldots, M\} \backslash\left\{j_{i}\right\} .
$$

Otherwise, the gradient of $G D_{p}$ is not defined at $A$. If condition (9) is satisfied, then (7) can be written as follows:

$$
G D_{p}(A):=\left(\frac{1}{N} \sum_{i=1}^{N}\left\|F\left(a_{i}\right)-z_{j_{i}}\right\|^{p}\right)^{\frac{1}{p}},
$$

and for the special case $p=2$ we obtain

$$
G D_{2}^{2}(A):=\frac{1}{N} \sum_{i=1}^{N}\left\|F\left(a_{i}\right)-z_{j_{i}}\right\|_{2}^{2} \in \mathbb{R}^{n \cdot N} .
$$


The gradient of $G D_{2}^{2}$ at $A$ is hence given by

$$
\nabla G D_{2}^{2}(A):=\frac{2}{N}\left(\begin{array}{c}
J\left(a_{1}\right)^{T}\left(F\left(a_{1}\right)-z_{j_{1}}\right) \\
J\left(a_{2}\right)^{T}\left(F\left(a_{2}\right)-z_{j_{2}}\right) \\
\vdots \\
J\left(a_{N}\right)^{T}\left(F\left(a_{N}\right)-z_{j_{N}}\right)
\end{array}\right) \in \mathbb{R}^{n \cdot N},
$$

where $J\left(a_{i}\right)$ denotes the Jacobian matrix of $F$ at $a_{i}$ for $i=1, \ldots, N$. We call the vector

$$
J\left(a_{i}\right)^{T}\left(F\left(a_{i}\right)-z_{j_{i}}\right), \quad i \in\{1, \ldots, N\},
$$

the $i$-th sub-gradient ( The sub-gradient is defined here as part of the gradient that is associated to an element $a$ of $A$, and is not equal to the notion of the sub-gradient known in non-smooth optimization. ) of $G D_{2}^{2}$ with respect to $a_{i} \in A$. Note that the sub-gradients are completely independent of the location of the other archive elements $a_{j} \in A$.

If the given MOP is unconstrained, then the first order necessary condition for optimality is that the gradient of $G D_{2}^{2}$ vanishes. This is the case for a set $A$ if all sub-gradients vanish

$$
\nabla G D_{2}^{2}(A)=0 \Leftrightarrow J\left(a_{i}\right)^{T}\left(F\left(a_{i}\right)-z_{j_{i}}\right)=0 \forall i=1, \ldots, N .
$$

This happens if for each $a_{i}$ either

(i) $F\left(a_{i}\right)=z_{j_{i}}$, that is, if the image of $a_{i}$ is equal to one of the elements of the reference set. This is for instance never the case if $Z$ is chosen utopian.

(ii) If $F\left(a_{i}\right) \neq z_{j_{i}}$, we have

$$
J\left(a_{i}\right)^{T}\left(F\left(a_{i}\right)-z_{j_{i}}\right)=\sum_{l=1}^{k} \nabla f_{l}\left(a_{i}\right) \underbrace{\left(f_{l}\left(a_{i}\right)-\left(z_{j_{i}}\right)_{l}\right)}_{=: \alpha_{l}^{(i)}}=\sum_{l=1}^{k} \alpha_{l}^{(i)} \nabla f_{l}\left(a_{i}\right)=0
$$

for a vector $\alpha^{(i)} \in \mathbb{R}^{k} \backslash\{0\}$. The point $a_{i}$ is hence a critical point since $\operatorname{rank}\left(J\left(a_{i}\right)\right)<k$. Furthermore, if $F\left(a_{i}\right)-z_{j_{i}} \geq_{p} 0$ (e.g., if $Z$ is again utopian) then $a_{i}$ is even a Karush-Kuhn-Tucker point. See Figure 2 for a geometrical interpretation of this scenario.

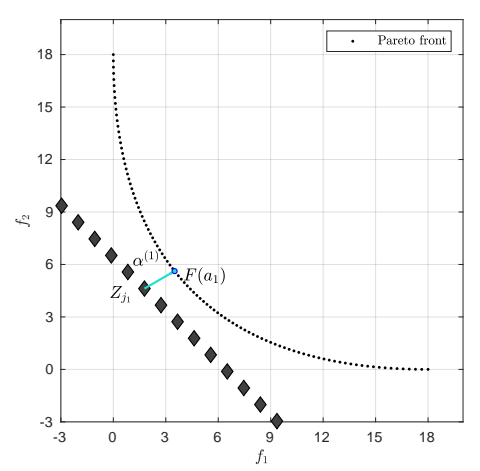

(a)

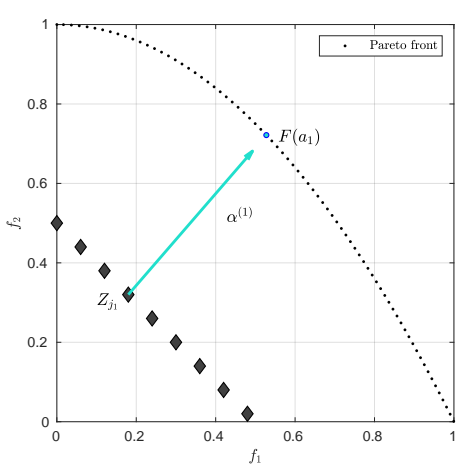

(b)

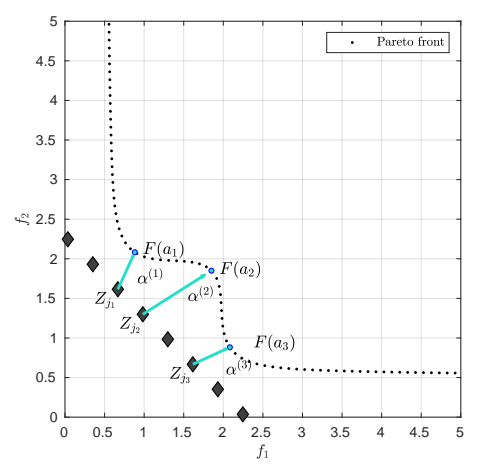

(c)

Figure 2. Geometrical interpretation of the optimality condition (ii) for $G D_{2}^{2}$. Note that $\alpha$ is orthogonal to the linearized Pareto front. (a) shows this behavior on a concave Pareto front, (b) on a convex Pareto front, and (c) on a concave/convex Pareto front. 


\subsubsection{Hessian of $G D_{2}^{2}$}

We first define the map $g: \mathbb{R}^{n} \rightarrow \mathbb{R}^{n}$ as

$$
g\left(a_{i}\right):=\sum_{l=1}^{k} \alpha_{l}^{(i)} \nabla f_{l}\left(a_{i}\right)
$$

where $\alpha^{(i)}$ is as in (15). In order to find an expression of the Hessian matrix, we now derive Equation (16) as follows:

$$
\mathcal{D} g\left(a_{i}\right)=\sum_{l=1}^{k}\left(\nabla f_{l}\left(a_{i}\right) \nabla f_{l}\left(a_{i}\right)^{T}+\alpha_{l} \nabla^{2} f_{l}\left(a_{i}\right)\right)=J\left(a_{i}\right)^{T} J\left(a_{i}\right)+W_{\alpha}\left(a_{i}\right) \in \mathbb{R}^{n \times n},
$$

where

$$
W_{\alpha}\left(a_{i}\right)=\sum_{l=1}^{k} \alpha_{l} \nabla^{2} f_{l}\left(a_{i}\right)
$$

Thus, the Hessian matrix of $G D_{2}^{2}$ is

$$
\nabla^{2} G D_{2}^{2}(A)=\frac{2}{N} \operatorname{diag}\left(\mathcal{D} g\left(a_{1}\right), \ldots, \mathcal{D} g\left(a_{N}\right)\right) \in \mathbb{R}^{n \cdot N \times n \cdot N},
$$

which is a block diagonal matrix.

\subsection{Gradient and Hessian for General $p>1$}

As mentioned above, we focus here on the special case $p=2$. The above derivatives, however, can be generalized for $p>1$ as follows (assuming that $Z$ is an utopian finite set to avoid problems when $p<4$ ): the gradient is given by

$$
\nabla G D_{p}^{p}(A):=\frac{p}{N}\left(\begin{array}{c}
\left\|F\left(a_{1}\right)-z_{j_{1}}\right\|^{p-2} J\left(a_{1}\right)^{T}\left(F\left(a_{1}\right)-z_{j_{1}}\right) \\
\left\|F\left(a_{2}\right)-z_{j_{2}}\right\|^{p-2} J\left(a_{2}\right)^{T}\left(F\left(a_{2}\right)-z_{j_{2}}\right) \\
\vdots \\
\left\|F\left(a_{N}\right)-z_{j_{N}}\right\|^{p-2} J\left(a_{N}\right)^{T}\left(F\left(a_{N}\right)-z_{j_{N}}\right)
\end{array}\right) \in \mathbb{R}^{n \cdot N},
$$

and the Hessian by

$$
\nabla^{2} G D_{p}^{p}(A)=\operatorname{diag}\left(\mathcal{H}_{1}, \ldots, \mathcal{H}_{N}\right) \in \mathbb{R}^{n \cdot N \times n \cdot N}
$$

where

$$
\begin{aligned}
\mathcal{H}_{i}= & \frac{p(p-2)}{N}\left\|F\left(a_{i}\right)-z_{j_{i}}\right\|^{p-4}\left[J\left(a_{i}\right)^{T}\left(F\left(a_{i}\right)-z_{j_{i}}\right)\left(F\left(a_{i}\right)-z_{j_{i}}\right)^{T} J\left(a_{i}\right)^{T}\right] \\
& +\frac{p}{N}\left[J\left(a_{i}\right)^{T} J\left(a_{i}\right)+W_{\alpha}\left(a_{i}\right)\right],
\end{aligned}
$$

for $i=1,2, \ldots, N$.

\section{3. $G D_{2}^{2}$-Newton Method}

After having derived the gradient and the Hessian we are now in the position to state the set based Newton method for the $G D_{2}^{2}$ indicator: 


\section{$G D_{2}^{2}$ Newton method}

$$
A^{0} \subset \mathbb{R}^{n}
$$

The Newton iteration can in practice be stopped at a set $A^{f}$ if

$$
\left\|\nabla G D_{2}^{2}\left(A^{f}\right)\right\| \leq t o l,
$$

for a given tolerance tol $>0$. In order to speed up the computations one may proceed due to the structure of the (sub-)gradient as follows: for each element $a_{i}$ of a current archive $A$ with

$$
\left\|J\left(a_{i}\right)^{T}\left(F\left(a_{i}\right)-z_{j_{i}}\right)\right\| \leq \frac{t o l}{\sqrt{N}}
$$

one can continue the Newton iteration with the smaller set $\bar{A}=A \backslash\left\{a_{i}\right\}$ (and later insert $a_{i}$ into the final archive).

We are particularly interested in the regularity of $\nabla^{2} G D_{2}^{2}$ at the optimal set, i.e., at a set $A^{*}$ that solves problem (5) for $I=G D_{2}^{2}$. This is the case since if the Hessian is regular at $A^{*}$-and if the objective function is sufficiently smooth-we can expect the Newton method to converge locally quadratically [51].

Since the Hessian is a block diagonal matrix it is regular if all of its blocks

$$
J\left(a_{i}\right)^{T} J\left(a_{i}\right)+W_{\alpha(i)}\left(a_{i}\right), \quad i=1, \ldots, N,
$$

are regular. From this we see already that if $Z$ is not utopian, we cannot expect quadratic convergence: assume that one point $z \in Z$ is feasible, i.e., that there exists one $x \in Q$ such that $F(x)=z$. We can assume that $x$ is also a member of the optimal set $A^{*}$, say $a_{i}=x$. Then, we have that the weight vector $\alpha^{(i)}$ is zero, and hence that $W_{\alpha^{(i)}}=\sum_{l=1}^{k} \alpha_{l}^{(i)} \nabla^{2} f_{l}\left(a_{i}\right)=0$. Thus, the block matrix reduces to $J\left(a_{i}\right)^{T} J\left(a_{i}\right)$ those rank is at most $k$. The block matrix is hence singular, and so is the Hessian of $G D_{2}^{2}$ at $A^{*}$.

In the case all individual objectives are strictly convex, the $G D_{2}^{2}$ Hessian is positive definite (and hence regular) at every feasible set $A$, and we can hence expect local quadratic convergence.

Proposition 1. Let a MOP of the form (2) be given whose individual objectives are strictly convex, and let $\mathrm{Z}$ be a discrete utopian set. Then, the matrix $\nabla^{2} G D_{2}^{2}(A)$ is positive definite for all feasible sets $A$.

Proof. Since $\nabla^{2} G D_{2}^{2}(A)$ is block diagonal, it is sufficient to consider the block matrices $J\left(a_{i}\right)^{T} J\left(a_{i}\right)+$ $W_{\alpha^{(i)}}\left(a_{i}\right), \quad i=1, \ldots, N$. Let $i \in\{1, \ldots, N\}$. Since $Z$ is utopian, it is $\alpha^{(i)} \neq 0$, and all of its elements are non-negative. Further, since all individual objectives $f_{l}$ are strictly convex, the matrices $\nabla^{2} f_{l}\left(a_{i}\right)$ are positive definite, and hence also the matrix $W_{\alpha}\left(a_{i}\right)$. Since $J^{T}\left(a_{i}\right) J\left(a_{i}\right)$ is positive semi-definite, we have for all $x \in \mathbb{R}^{n} \backslash\{0\}$

$$
x^{T}\left(J\left(a_{i}\right)^{T} J\left(a_{i}\right)+W_{\alpha^{(i)}}\right) x=x^{T} J\left(a_{i}\right)^{T} J\left(a_{i}\right) x+x^{T} W_{\alpha^{(i)}} x>0,
$$

since $x^{T} J\left(a_{i}\right)^{T} J\left(a_{i}\right) x \geq 0$ and $x^{T} W_{\alpha^{(i)}} x>0$. Therefore, each $\mathcal{D} g\left(a_{i}\right), i=1, \ldots, N$, is positive definite and hence also the matrix $\nabla^{2} G D_{2}^{2}(A)$. 


\subsection{Example}

We consider the following convex bi-objective problem

$$
\begin{aligned}
& f_{1}, f_{2}: \mathbb{R}^{2} \rightarrow \mathbb{R} \\
& f_{1}(x)=x_{1}^{2}+\left(x_{2}+3\right)^{2} \\
& f_{2}(x)=\left(x_{1}+3\right)^{2}+x_{2}^{2}
\end{aligned}
$$

Figure 3 shows the Pareto front of this problem together with the reference set $Z$ that contains 30 elements (black dots). The set $Z$ is a discretization of the convex hull of individual minima (CHIM, [23]) of the problem that has been shifted left down. Further, it shows the images of the Newton steps of an initial set $A_{0}$ that contains 21 elements. As it can be seen, all images converge toward three solutions that are placed in the middle of the Pareto front (which is owed to the fact that $Z$ is discrete. If $Z$ would be continuous, all images would converge toward one solution). This example already shows that the $G D_{2}^{2}$ Newton method is of restricted interest as standalone algorithm. The method will, however, become important as part of the $\Delta_{p}$-Newton method as it will become apparent later on. Table 1 shows the respective $G D_{2}^{2}$ values plus the norms of the gradients which indicate quadratic convergence. The second column indicates that the images of the archives converge toward the Pareto front as anticipated.
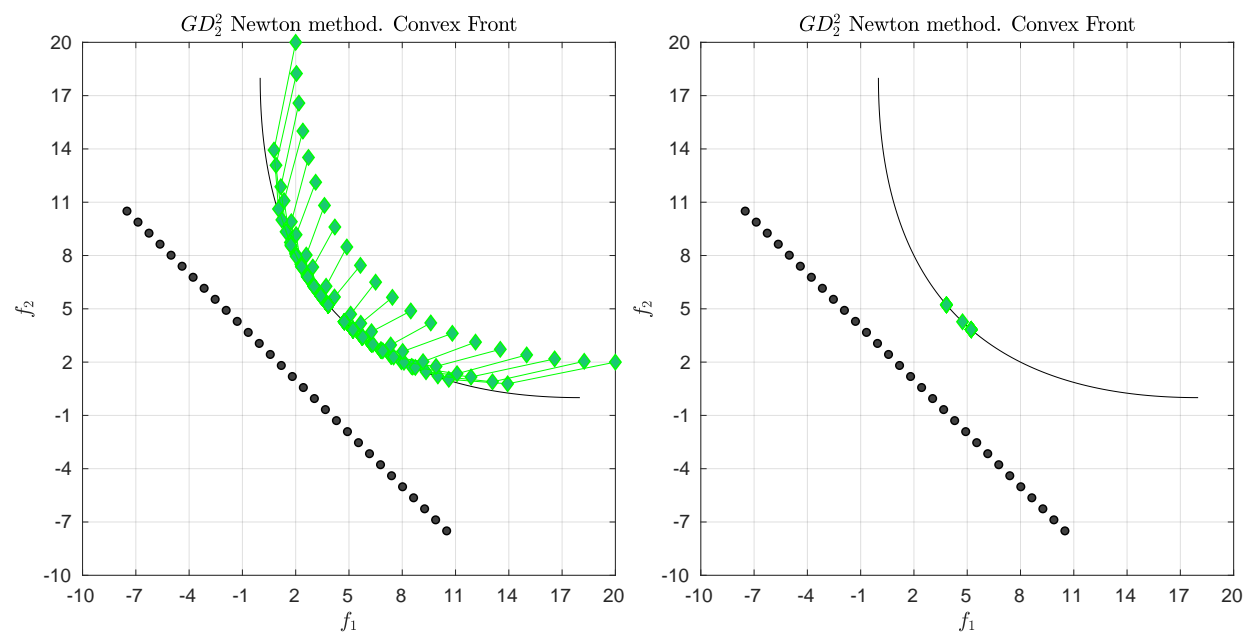

Figure 3. (Left) application of the $G D_{2}^{2}$ Newton method on bi-objective oriented problem (BOP) (27). (Right) only the final archive is shown.

Table 1. Numerical results of the $G D_{2}^{2}$-Newton method for BOP (27).

\begin{tabular}{cccc}
\hline Iter. & $\left\|\nabla G D_{2}^{2}\left(A^{i}\right)\right\|$ & $G D_{2}^{2}\left(F\left(A^{i}\right), F\left(P_{Q}\right)\right)$ & $G D_{2}^{2}\left(F\left(A^{i}\right), Z\right)$ \\
\hline 0 & - & 12.000000000000000 & 2.102524077758237 \\
1 & 24.575789798441914 & 2.443855313744088 & 1.364160070353236 \\
2 & 10.174108923911083 & 0.155893831541973 & 1.099322040004995 \\
3 & 5.003263195893473 & 0.002209872937986 & 1.014751905633911 \\
4 & 3.169714351377499 & 0.000015254816873 & 0.976329099745630 \\
5 & 1.947602617177173 & 0.000000021343544 & 0.957865673825140 \\
6 & 1.758375206901766 & 0.000000000020256 & 0.945790145414235 \\
7 & 1.433193382521511 & 0.000000000000013 & 0.939274242767051 \\
8 & 1.012249366157551 & 0.000000000000000 & 0.936469149315602 \\
9 & 0.006408088020990 & 0.000000000000000 & 0.936469035893491 \\
10 & 0.000000182419413 & 0 & 0.936469035893491 \\
11 & 0.000000000000002 & 0 & 0.936469035893491 \\
\hline
\end{tabular}




\section{4. $I G D_{p}$ Newton Method}

The indicator $I G D_{p}$ computes how far, on average, the discrete reference set $Z$ is from a given archive $A$, and is defined as

$$
\operatorname{IGD}(A):=\left(\frac{1}{M} \sum_{i=1}^{M} d\left(z_{i}, F(A)\right)^{p}\right)^{\frac{1}{p}}
$$

where $d\left(z_{i}, F(A)\right)$ is given by

$$
d\left(z_{i}, F(A)\right):=\min _{j=1, \ldots, N}\left\|z_{i}-F\left(a_{j}\right)\right\|, \text { for } i=1, \ldots, M .
$$

\subsection{Gradient of $I G D_{p}$}

Similar to $G D$, we will also have to assume that for all $i=1, \ldots, M$ there exists an index $j_{i} \in\{1, \ldots, N\}$ such that:

$$
d\left(z_{i}, F(A)\right)=\left\|z_{i}-F\left(a_{j_{i}}\right)\right\|<\left\|z_{i}-F\left(a_{q}\right)\right\| \forall q \in\{1, \ldots, N\} \backslash\left\{j_{i}\right\},
$$

since otherwise the gradient of $I G D_{p}$ is not defined. Then, using Equation (30), Equation (28) can be written as follows:

$$
\operatorname{IGD}_{p}(A):=\left(\frac{1}{M} \sum_{i=1}^{M}\left\|z_{i}-F\left(a_{j_{i}}\right)\right\|^{p}\right)^{\frac{1}{p}} .
$$

From now on we will consider $I G D_{2}^{2}$ which is given by

$$
\operatorname{IGD} D_{2}^{2}(A):=\frac{1}{M} \sum_{i=1}^{M}\left\|z_{i}-F\left(a_{j_{i}}\right)\right\|_{2}^{2}
$$

In order to derive the gradient of $I G D_{2}^{2}$, let $I_{l}:=\left\{i: j_{i}=l\right\}, l \in\{1, \ldots, N\}$, be the set formed by the indexes $i \in\{1,2, \ldots, M\}$ that are related to $j_{i}$. In other words, this set gives us the relation of the elements of $Z$ related to each image $F\left(a_{l}\right)$ (an example of this relation can be found in Figure 4). Then, the sub-gradient of $I G D_{2}^{2}$ at point $a_{l}$ is given by

$$
\frac{\partial I G D_{2}^{2}}{\partial a_{l}}(A)=\frac{2}{M} \sum_{i \in I_{l}} J\left(a_{l}\right)^{T}\left(F\left(a_{l}\right)-z_{i}\right)=\frac{2}{M} J\left(a_{l}\right)^{T}\left(m_{l} F\left(a_{l}\right)-\sum_{i \in I_{l}} z_{i}\right),
$$

where $m_{l}=\left|I_{l}\right|$. Finally, the gradient of $I G D_{2}^{2}$ can be expressed as

$$
\nabla I G D_{2}^{2}(A):=\left(\begin{array}{c}
\frac{\partial I G D_{2}^{2}}{\partial a_{1}}(A) \\
\frac{\partial I G D_{2}^{2}}{\partial a_{2}}(A) \\
\vdots \\
\frac{\partial I G D_{2}^{2}}{\partial a_{N}}(A)
\end{array}\right) \in \mathbb{R}^{n \cdot N}
$$

It is worth to notice that the sub-gradients depend on the location of the other archive elements which implies a "group motion" (which is in contrast to the gradient of $G D_{2}^{2}$ ). 


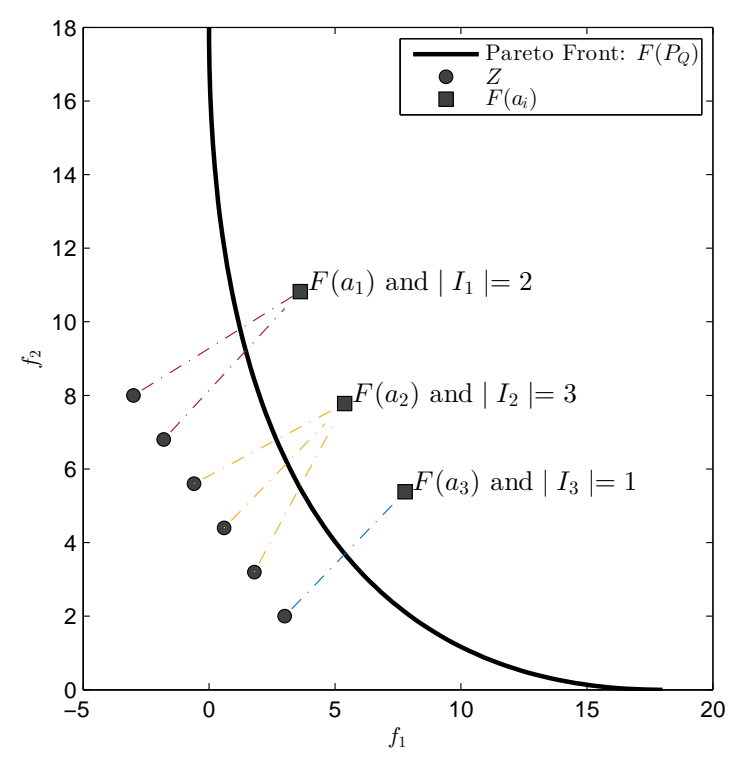

Figure 4. Example of a relation between the reference set $Z$ and the approximation set $F(A)$.

We next consider under which conditions the gradient of $I G D_{2}^{2}$ vanishes. If $\nabla \operatorname{IGD}_{2}^{2}(A)=0$, then for all $l=1, \ldots, N$ we have that

$$
\begin{aligned}
& J\left(a_{l}\right)^{T}\left(m_{l} F\left(a_{l}\right)-\sum_{i \in I_{l}} z_{i}\right)=0 \\
& \Leftrightarrow J\left(a_{l}\right)^{T} F\left(a_{l}\right)=J\left(a_{l}\right)^{T} \underbrace{\left(\frac{\sum_{i \in I_{l}} z_{i}}{m_{l}}\right)}_{C_{l}},
\end{aligned}
$$

where $C$ is the centroid of $z_{i}{ }^{\prime}$ s. Then, note that if:

1. $\operatorname{rank}\left(J\left(a_{l}\right)\right)=k$, then $F\left(a_{l}\right)=\frac{\sum_{i \in I_{l}} z_{i}}{m_{l}}=C_{l}$.

2. $\operatorname{rank}\left(J\left(a_{l}\right)\right)=k-1$, then $F\left(a_{l}\right)-C_{l}$ is orthogonal to the linearized image of $F$ at $F\left(a_{l}\right)$, and orthogonal to the linearized Pareto front at $F\left(a_{l}\right)$ in case $F\left(a_{l}\right)-C_{l} \geq_{p} 0$ and $F\left(a_{l}\right)-C_{l} \neq 0$ (see Figure 5 for such a scenario).

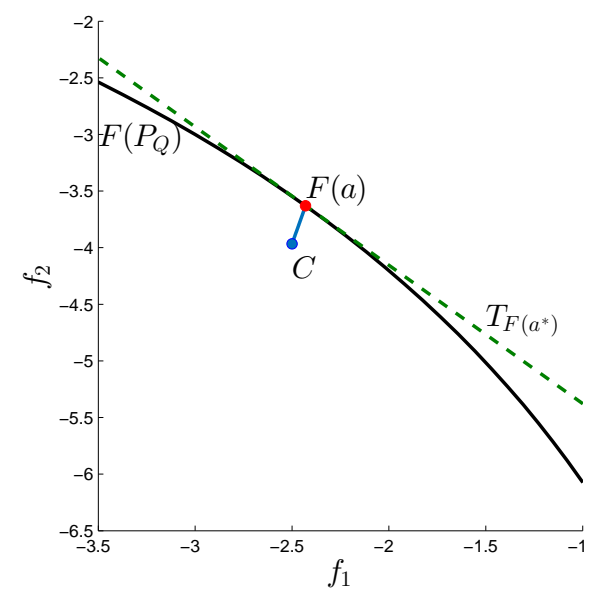

Figure 5. Geometric interpretation when $F\left(a_{l}\right)-C$ is orthogonal to the linearized Pareto front. 
4.2. Hessian Matrix of $I G D_{p}$

Analog to the derivation of $G D_{p}$ - Hessian, we first define the map $g: \mathbb{R}^{n} \rightarrow \mathbb{R}^{n}$ as

$$
g\left(a_{l}\right):=J\left(a_{l}\right)^{T}\left(m_{l} F\left(a_{l}\right)-\sum_{i \in I_{l}} z_{i}\right) .
$$

Now, let $\sum_{i \in I_{l}} z_{i}=y=\left(y 1, \ldots, y_{k}\right)^{T}$. Then

$$
g\left(a_{l}\right)=J\left(a_{l}\right)^{T}\left(m_{l} F\left(a_{l}\right)-y\right)=m_{l} \sum_{i=1}^{k} f_{i}(x) \nabla f_{i}(x)-\sum_{i=1}^{k} y_{i} \nabla f_{i}(x) .
$$

Then, we derive Equation (38) as follows:

$$
\begin{aligned}
\mathcal{D} g\left(a_{l}\right) & =m_{l} \sum_{i=1}^{k} f_{i}\left(a_{l}\right) \nabla^{2} f_{i}\left(a_{l}\right)+m_{l} J\left(a_{l}\right)^{T} J\left(a_{l}\right)-\sum_{i=1}^{k} y_{i} \nabla^{2} f_{i}\left(a_{l}\right) \\
& =\sum_{i=1}^{k}\left(m_{l} f_{i}\left(a_{l}\right)-y_{i}\right) \nabla^{2} f_{i}\left(a_{l}\right)+m_{l} J\left(a_{l}\right)^{T} J\left(a_{l}\right) \\
& =m_{l} J\left(a_{l}\right)^{T} J\left(a_{l}\right)+W_{\alpha}\left(a_{l}\right) \in \mathbb{R}^{n \times n},
\end{aligned}
$$

where

$$
W_{\alpha}\left(a_{l}\right)=\sum_{i=1}^{k} \underbrace{\left(m_{l} f_{i}\left(a_{l}\right)-y_{i}\right)}_{:=\alpha_{i}^{(l)}} \nabla^{2} f_{i}\left(a_{l}\right)=\sum_{i=1}^{k} \alpha_{i}^{(l)} \nabla^{2} f_{i}\left(a_{l}\right) .
$$

Thus, the Hessian matrix of $I G D_{2}^{2}$ is given by

$$
\nabla^{2} I G D_{2}^{2}(A)=\frac{2}{M} \operatorname{diag}\left(\mathcal{D} g\left(a_{1}\right), \ldots, \mathcal{D} g\left(a_{N}\right)\right) \in \mathbb{R}^{n \cdot N \times n \cdot N},
$$

which is a block diagonal matrix.

\subsection{Gradient and Hessian for General $p>1$}

The above derivatives can be generalized for $p>1$ as follows: the gradient is given by

$$
\nabla \operatorname{IG} D_{p}^{p}(A):=\frac{p}{M}\left(\begin{array}{c}
J\left(a_{1}\right)^{T} \sum_{i \in I_{1}}\left\|F\left(a_{1}\right)-z_{i}\right\|^{p-2}\left(F\left(a_{1}\right)-z_{i}\right) \\
J\left(a_{2}\right)^{T} \sum_{i \in I_{2}}\left\|F\left(a_{2}\right)-z_{i}\right\|^{p-2}\left(F\left(a_{2}\right)-z_{i}\right) \\
\vdots \\
J\left(a_{N}\right)^{T} \sum_{i \in I_{N}}\left\|F\left(a_{N}\right)-z_{i}\right\|^{p-2}\left(F\left(a_{N}\right)-z_{i}\right)
\end{array}\right) \in \mathbb{R}^{n \cdot N}
$$

and the Hessian by

$$
\nabla^{2} I G D_{p}^{p}(A)=\operatorname{diag}\left(\mathcal{H}_{1}, \ldots, \mathcal{H}_{N}\right) \in \mathbb{R}^{n \cdot N \times n \cdot N},
$$

where

$$
\begin{aligned}
\mathcal{H}_{l}= & \frac{p(p-2)}{N} \sum_{i \in I_{l}}\left\|F\left(a_{l}\right)-z_{i}\right\|^{p-4}\left[J\left(a_{l}\right)^{T}\left(F\left(a_{l}\right)-z_{i}\right)\left(F\left(a_{l}\right)-z_{i}\right)^{T} J\left(a_{l}\right)^{T}\right] \\
& +\frac{p}{M}\left[J\left(a_{l}\right)^{T} J\left(a_{l}\right)+W_{\alpha}\left(a_{l}\right)\right] .
\end{aligned}
$$




\subsection{IGD $D_{2}^{2}$ Newton Method}

After having derived the gradient and the Hessian of $I G D_{2}^{2}$ we are now in the position to state the respective set based Newton method.

$I G D_{2}^{2}$ Newton method

$$
A^{0} \subset \mathbb{R}^{n}:=A^{t}-\operatorname{diag}\left(\mathcal{D} g\left(a_{1}\right), \ldots, \mathcal{D} g\left(a_{N}\right)\right)^{-1}\left(\begin{array}{c}
J\left(a_{1}\right)^{T}\left(m_{1} F\left(a_{1}\right)-\sum_{i \in I_{1}} z_{i}\right) \\
\vdots \\
J\left(a_{N}\right)^{T}\left(m_{N} F\left(a_{N}\right)-\sum_{i \in I_{N}} z_{i}\right)
\end{array}\right), \quad t=0,1, \ldots
$$

Similarly to the GD Newton method, the iteration can be stopped at an set $A^{f}$ if

$$
\left\|\nabla I G D_{2}^{2}\left(A^{f}\right)\right\| \leq t o l
$$

for a given tolerance $t o l>0$, and the iteration for an element $a_{i}$ can be stopped when

$$
\frac{2}{M} J\left(a_{l}\right)^{T}\left(m_{l} F\left(a_{l}\right)-\sum_{i \in I_{l}} z_{i}\right) \leq \frac{t o l}{\sqrt{N}}
$$

One important special case is when the image $F\left(a_{l}\right)$ of a point $a_{l}$ of a set $A$ is not the nearest point of any element of $Z$, i.e., if $m_{l}=0$ (see Figure 6). In that case, the $l$-th sub-gradient vanishes,

$$
m_{l}=0 \Rightarrow \frac{\partial I G D_{2}^{2}}{\partial a_{l}}(A)=0,
$$

which means that the point $a_{l}$ will remain fixed under further iterations of the IGD Newton method. One possibility is hence to neglect such points in subsequent iterations, and to continue with the reduced set. Note also that dominance and distance are two different concepts. That is, if all points of a set $A$ are mutually non-dominated, this does give an implication on $m_{l}$, see Figure 7 for two examples.

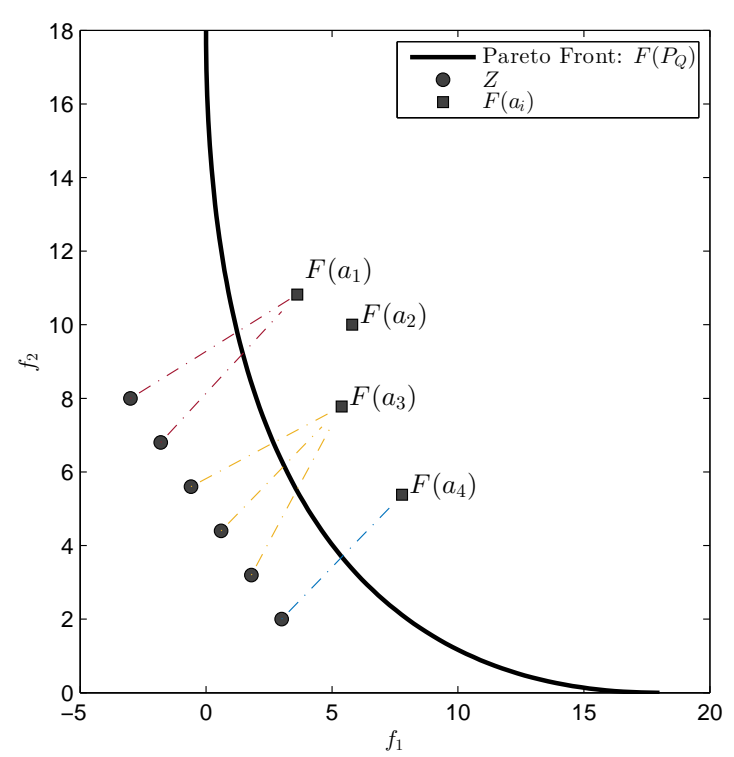

Figure 6. Potential problem of the Inverted Generational Distance (IGD) Newton method: if $m_{l}=0$ (here it is $m_{2}=0$ ) then the $l$-th sub-gradient is equal to zero, and $a_{l}$ will stay fixed under the Newton iteration. 

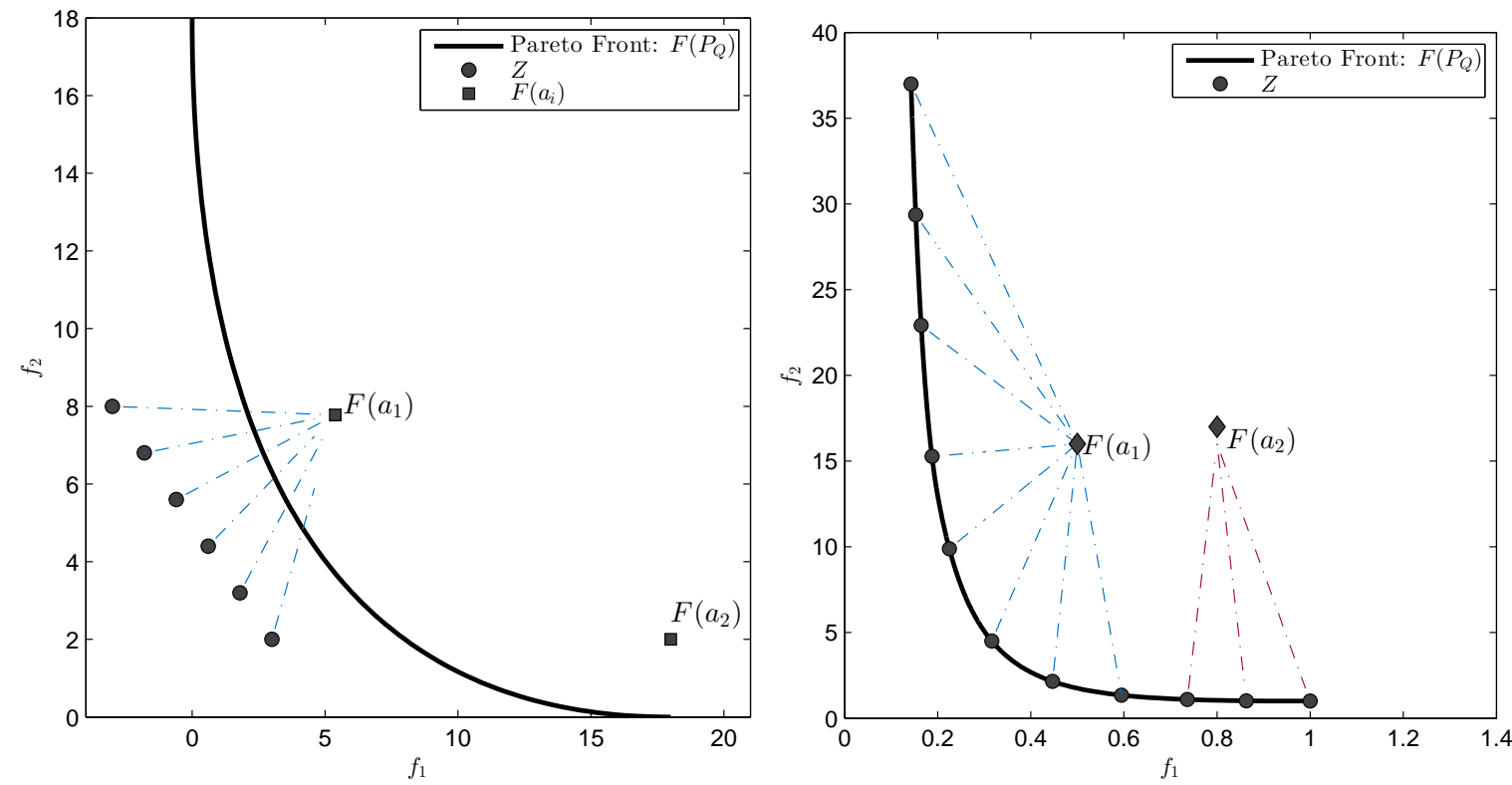

Figure 7. Dominance and distance are different concepts. (Left) an example where $a_{1}$ and $a_{2}$ are mutually non-dominated, but where $\left|I_{2}\right|=0$. (Right) an example where $a_{1} \prec a_{2}$, but $\left|I_{l}\right| \neq 0$ for $l \in\{1,2\}$.

Similar to GD, we are interested in the regularity of $\nabla^{2} I G D_{2}^{2}$ at the optimal set since we can in that case expect local quadratic convergence. By the structure of the Hessian we have singularity in the following cases:

1. if $m_{l}=0$ for a $l \in\{1, \ldots, N\}$ (since then $\mathcal{D} g\left(a_{l}\right)=0$ ) (see also the discussion above), and

2. if one element $z_{l}$ of $Z$ is feasible (since then $\mathcal{D} g\left(a_{l}\right)=J\left(a_{l}\right)^{T} J\left(a_{l}\right)$ which has a rank $\leq k$, and under the assumption that $k<n$ ).

Similar as for $G D$, the IGD-Hessian is positive definite for strictly convex problems and utopian reference sets if in addition $m_{l} \geq 1$ for all $l \in\{1, \ldots, N\}$.

Proposition 2. Let a MOP of the form (2) be given whose individual objectives are strictly convex, and let $Z$ be a discrete utopian set. Further, let $m_{l} \geq 1$ for all $l \in\{1, \ldots, N\}$, and $A$ be feasible. Then, the matrix $\nabla^{2} I G D_{2}^{2}(A)$ is positive definite.

Proof. Let $l \in\{1, \ldots, N\}$, and for ease of notation $\bigcup_{i \in I_{l}} z_{i}=\left\{Z^{1}, \ldots, Z^{m_{l}}\right\}$. Since all $Z^{i \prime}$ s are utopian we have

$$
\alpha^{(l)}=m_{l} F\left(a_{l}\right)-\sum_{i \in I_{l}} z_{i}=\underbrace{F\left(a_{l}\right)-Z^{1}}_{\geq_{p} 0}+\cdots+\underbrace{F\left(a_{l}\right)-Z^{m_{l}}}_{\geq_{p} 0} \geq_{p} 0,
$$

as well as $m_{l} F\left(a_{l}\right) \neq \sum_{i \in I_{l}} z_{i}$ (and hence $\alpha^{(l)} \neq 0$ ). The rest is analog to the proof of Proposition 1 .

\subsection{Examples}

We first consider again the convex BOP (27) as for the previous example (see Figure 8), but now using the IGD-Newton method. Already after one iteration step for 8 out of the 21 elements it is $m_{l}=0$ (denoted by red dots), and we continue the Newton method with the resulting 13-element subset. For this, we obtain quadratic convergence toward the ideal set (for $N=13$ ) as it can be observed from Table 2 . 

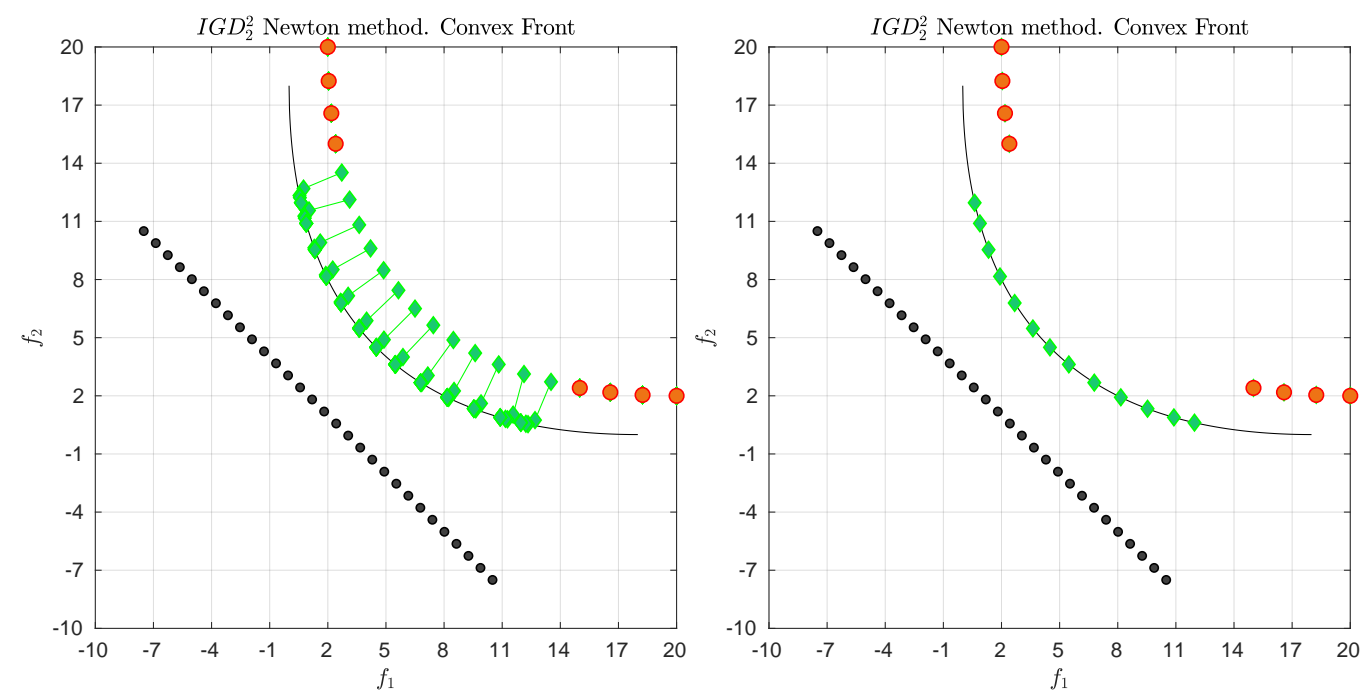

Figure 8. (Left) application of the $I G D_{2}^{2}$-Newton method on BOP (27). (Right) image of the final archive (green) together with the images for those $m_{l}=0$ (red).

Table 2. Numerical results of $I G D_{2}^{2}$-Newton method for BOP (27), see Figure 8.

\begin{tabular}{cccc}
\hline Iter. & $\left\|\nabla I G D_{2}^{2}\left(A^{i}\right)\right\|$ & $G D_{2}^{2}\left(F\left(A^{i}\right), F\left(P_{Q}\right)\right)$ & $I G D_{2}^{2}\left(F\left(A^{i}\right), Z\right)$ \\
\hline 0 & - & 12.000000000000000 & 0.280604068205798 \\
1 & 18.378420484981000 & 1.930506027522264 & 0.206869895378755 \\
2 & 5.432039146770605 & 0.043471951768718 & 0.192756253890335 \\
3 & 0.817043487084936 & 0.000003701993633 & 0.192391225092683 \\
4 & 0.706420510642436 & 0.000000000171966 & 0.192326368208389 \\
5 & 0.006184251273371 & 0.000000000000000 & 0.192326331507963 \\
6 & 0.000000481423311 & 0 & 0.192326331505474 \\
7 & 0.000000000000007 & 0 & 0.192326331505474 \\
\hline
\end{tabular}

We next consider the following BOP [52]

$$
\begin{aligned}
& f_{1}, f_{2}:[-4,4]^{2} \subset \mathbb{R}^{2} \rightarrow \mathbb{R} \\
& f_{1}(x)=1-\exp \left[-\left(x_{1}-\frac{1}{\sqrt{2}}\right)^{2}-\left(x_{2}-\frac{1}{\sqrt{2}}\right)^{2}\right] \\
& f_{2}(x)=1-\exp \left[-\left(x_{1}+\frac{1}{\sqrt{2}}\right)^{2}-\left(x_{2}+\frac{1}{\sqrt{2}}\right)^{2}\right]
\end{aligned}
$$

those Pareto front is concave. We apply the IGD-Newton method on the sets $A^{0}$ with $\left|A^{0}\right|=21$ and $Z$ with $|Z|=30$ as shown in Figure 9. For this example, only six elements of $A^{0}$ are closest to one element of $Z$. Table 3 shows that the convergence is much slower than for the previous example.

Finally, we consider the BOP [53]

$$
\begin{aligned}
f_{1}, f_{2} & : \mathbb{R}^{2} \rightarrow \mathbb{R} \\
f_{1}(x, y) & =\frac{1}{2}\left(\sqrt{1+(x+y)^{2}}+\sqrt{1+(x-y)^{2}}+x-y\right)+\lambda \cdot e^{-(x-y)^{2}} \\
f_{2}(x, y) & =\frac{1}{2}\left(\sqrt{1+(x+y)^{2}}+\sqrt{1+(x-y)^{2}}-x+y\right)+\lambda \cdot e^{-(x-y)^{2}}
\end{aligned}
$$


For $\lambda=0.85$ and $Q=[-1.5,1.5]^{2}$ the Pareto front containts a "dent" and is hence convex-concave. Figure 10 shows the setting and Table 4 the respective convergence behavior. Again, we "lose" elements from $A^{0}$ since for them there are no elements of $Z$ that are closest to them.
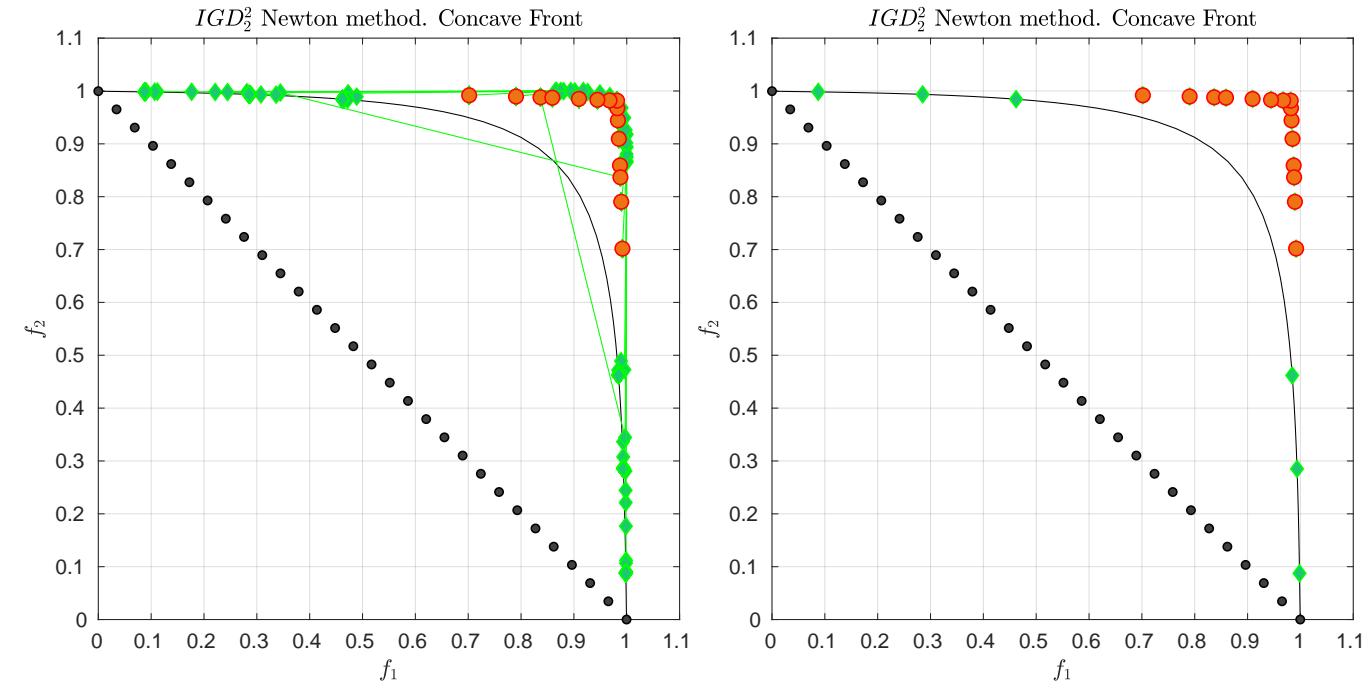

Figure 9. (Left) application of the $I G D_{2}^{2}$-Newton method on BOP (50). (Right) the image of the final archive (green) together with the images for which $m_{l}=0$.

Table 3. Numerical results of $I G D_{2}^{2}$-Newton method for BOP (50), see Figure 9.

\begin{tabular}{cccc}
\hline Iter. & $\left\|\nabla I G D_{2}^{2}\left(A^{i}\right)\right\|$ & $I G D_{2}^{2}\left(F\left(A^{i}\right), F\left(P_{Q}\right)\right)$ & $I G D_{2}^{2}\left(F\left(A^{i}\right), Z\right)$ \\
\hline 0 & - & 0.278373584606464 & 0.023220380628487 \\
1 & 0.336586538953659 & 0.201027590101253 & 0.008847986218368 \\
2 & 0.037592704195443 & 0.208694136517504 & 0.008453782829010 \\
3 & 0.020266018162657 & 0.205037496184976 & 0.008317268653462 \\
4 & 0.004947265003093 & 0.197436504168332 & 0.008331864711939 \\
5 & 0.012675095498115 & 0.196899482529974 & 0.008335126593931 \\
6 & 0.011342458560546 & 0.195934897889421 & 0.008256278024484 \\
7 & 0.001644428661330 & 0.194957155951972 & 0.008252791569886 \\
8 & 0.001355427544721 & 0.194529344670235 & 0.008248151529685 \\
9 & 0.000531420743367 & 0.194275287179155 & 0.008247144931520 \\
10 & 0.000462304446354 & 0.194197084382389 & 0.008244551897282 \\
11 & 0.000160142304221 & 0.194173320804311 & 0.008243923138606 \\
12 & 0.000083987735463 & 0.194171755397215 & 0.008243322467470 \\
13 & 0.000007989306735 & 0.194171718496160 & 0.008243262849450 \\
14 & 0.000000313502013 & 0.194171718485903 & 0.008243260421944 \\
15 & 0.000000004246450 & 0.194171718485903 & 0.008243260388981 \\
16 & 0.000000000077470 & 0.194171718485903 & 0.008243260388530 \\
17 & 0.000000000010284 & 0.194171718485903 & 0.008243260388522 \\
18 & 0.00000000001988 & 0.194171718485903 & 0.008243260388521 \\
19 & 0.000000000000385 & 0.194171718485903 & 0.008243260388521 \\
20 & 0.000000000000075 & 0.194171718485903 & 0.008243260388521 \\
\hline
\end{tabular}

Table 4. Numerical results of $I G D_{2}^{2}$-Newton method for BOP (51), see Figure 10.

\begin{tabular}{cccc}
\hline Iter. & $\left\|\nabla G D_{2}^{2}\left(A^{i}\right)\right\|$ & $I G D_{2}^{2}\left(F\left(A^{i}\right), F\left(P_{Q}\right)\right)$ & $I G D_{2}^{2}\left(F\left(A^{i}\right), Z\right)$ \\
\hline 0 & - & 0.743479945976417 & 0.089706026039859 \\
1 & 1.774223579432539 & 0.598423777888877 & 0.069797721774084 \\
2 & 1.059917072891813 & 0.580388865733755 & 0.065730197299576 \\
3 & 0.436102700877237 & 0.575606525856331 & 0.065186338106827 \\
4 & 0.044524401746943 & 0.575571396044490 & 0.065172539475108 \\
5 & 0.000368536791948 & 0.575571392056566 & 0.065172518364193 \\
6 & 0.000000023167599 & 0.575571392056566 & 0.065172518358461 \\
7 & 0.000000000000001 & 0.575571392056566 & 0.065172518358461 \\
\hline
\end{tabular}



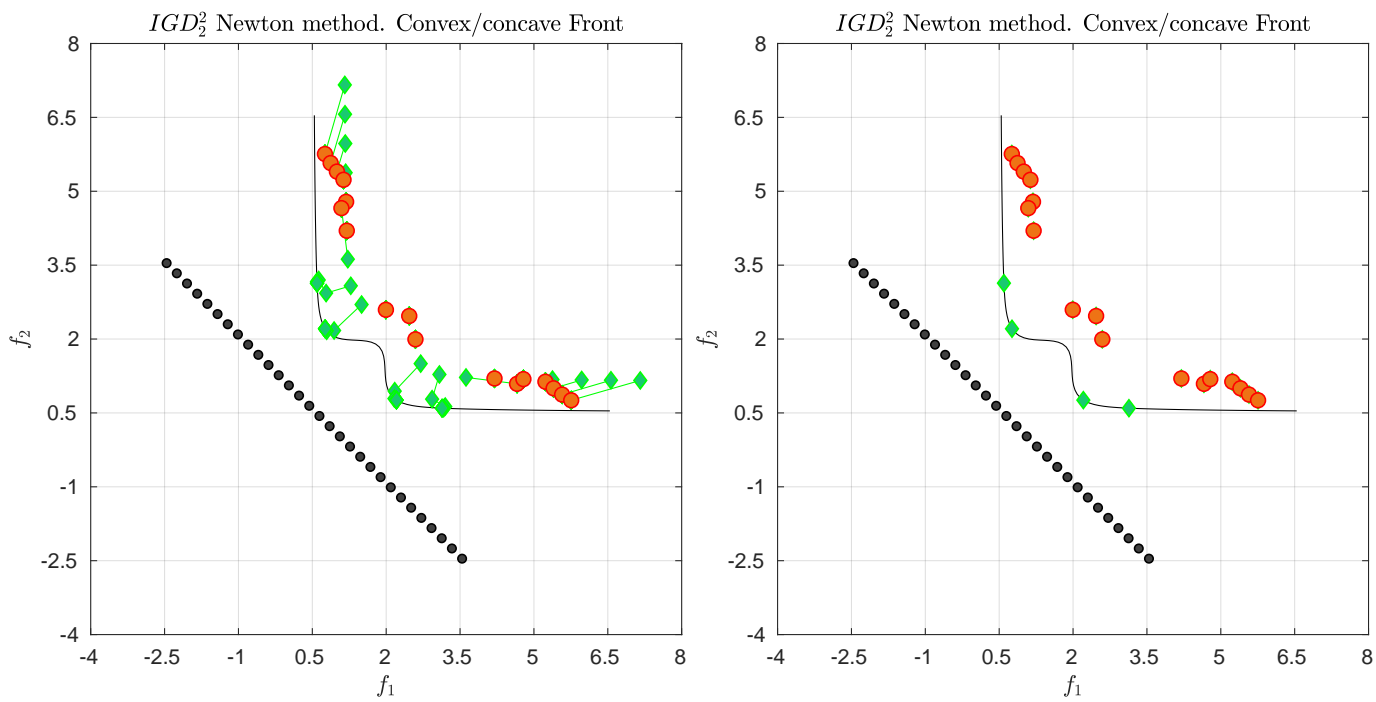

Figure 10. (Left) application of the $I G D_{2}^{2}$-Newton method on BOP (51). (Right) only the final archive is shown.

\section{5. $\Delta_{2}$-Newton Method}

Based on the results of the previous two sections we are now in the position to state the set based Newton method for the $\Delta_{2}^{2}$ indicator.

\section{1. $\Delta_{2}$-Newton Method}

Since $\Delta_{2}^{2}\left(A^{i}, Z\right)$ is defined by the maximum of $G D_{2}^{2}\left(A^{i}, Z\right)$ and $\operatorname{IGD}_{2}^{2}\left(A^{i}, Z\right)$ we simply check in each iteration which of the latter two values is larger, and apply either the GD or the IGD-Newton step accordingly.

$\Delta_{2}^{2}$-Newton method

$$
\begin{aligned}
& A^{0} \subset \mathbb{R}^{n} \\
& \text { for } i=0,1, \ldots \\
& \text { if } \quad G D_{2}^{2}\left(A^{i}, Z\right)>I G D_{2}^{2}\left(A^{i}, Z\right) \quad \text { (use } G D_{2}^{2} \text {-Newton step) } \\
& A^{t+1}:=A^{t}-\operatorname{diag}\left(\mathcal{D} g\left(a_{1}\right), \ldots, \mathcal{D} g\left(a_{N}\right)\right)^{-1}\left(\begin{array}{c}
J\left(a_{1}\right)^{T}\left(F\left(a_{1}\right)-z_{j_{1}}\right) \\
\vdots \\
J\left(a_{N}\right)^{T}\left(F\left(a_{N}\right)-z_{j_{N}}\right)
\end{array}\right) \\
& A^{t+1}:=A^{t}-\operatorname{diag}\left(\mathcal{D} g\left(a_{1}\right), \ldots, \mathcal{D} g\left(a_{N}\right)\right)^{-1}\left(\begin{array}{c}
J\left(a_{1}\right)^{T}\left(m_{1} F\left(a_{1}\right)-\sum_{i \in I_{1}} z_{i}\right) \\
\vdots \\
J\left(a_{N}\right)^{T}\left(m_{N} F\left(a_{N}\right)-\sum_{i \in I_{N}} z_{i}\right)
\end{array}\right)
\end{aligned}
$$

The properties and the realization of the method are in principle as for the $G D$ and the IGD-Newton method. The only difference, at least theoretically, is a possible loss of smoothness since $\Delta_{p}$ is defined by the maximum of two functions. Such issues, at least for convergence, however, are only to be expected in case $G D\left(A^{*}, Z\right)$ is equal to $\operatorname{IGD}\left(A^{*}, Z\right)$ for a reference set $Z$ and the respective optimal archive $A^{*}$. The cost for the realization one Newton step for each of the three indicators is $\mathrm{O}\left(\mathrm{Nn}^{2}\right)$ in storage when taking into account the block structure of the Hessians since $N$ matrices of dimension $n \times n$ have to be stored, and $O\left(N n^{3}\right)$ in terms of flops since $N$ linear equation systems of dimension $n$ have to be solved. 


\subsection{Examples}

We will in the following demonstrate the applicability of the $\Delta_{2}^{2}$-Newton method on several methods. For this, we first consider the same three examples and settings as for the $\operatorname{IGD}_{2}^{2}$-Newton method presented above. Figures 11-13 show some numerical results of the $\Delta_{2}$-Newton method using the same initial archives $A^{0}$ and reference sets $Z$ as in the previous section. As it can be seen, in all cases the method achieved much better approximations as for the sole usage of the IGD-Newton method (as well as the GD-Newton method). The convergence behaviors can be seen in Tables 5-7. In all cases, the GD value is the greater one in the initial steps of the method. After some iterations (and switches from GD to IGD and vice versa), however, the IGD value becomes eventually the largest one so that the $\Delta_{p}$-Newton method eventually coincides with the IGD-Newton method. In comparison to the results of the IGD-Newton method, however, it becomes apparent that the GD-Newton steps are in fact important to obtain better overall approximations.

Table 5. Numerical results of the $\Delta_{2}^{2}$-Newton method on BOP (27), see Figure 11.

\begin{tabular}{ccccl}
\hline Iter. & $\left\|\nabla \Delta_{2}^{2}\left(A^{i}\right)\right\|$ & $\Delta_{2}^{2}\left(F\left(A^{i}\right), F\left(P_{Q}\right)\right)$ & $\Delta_{2}^{2}\left(F\left(A^{i}\right), Z\right)$ & Indicator \\
\hline 0 & - & 2.000000000000000 & 2.102524077758237 & GD \\
1 & 24.575789798441914 & 0.410124138028425 & 1.364160070353236 & GD \\
2 & 10.174108923911083 & 0.026608923543674 & 1.099322040004995 & IGD \\
3 & 3.542645526228592 & 0.000015520657566 & 1.104000035194028 & GD \\
4 & 5.213757247004612 & 0.000000441436988 & 1.020226507943846 & IGD \\
5 & 9.260923965020773 & 0.000000016030350 & 1.036249331407054 & IGD \\
6 & 2.625118989394418 & 0.000000384599061 & 1.047878336296791 & IGD \\
7 & 0.042216669617238 & 0.000000384618980 & 1.047678945935868 & IGD \\
8 & 0.000010909557366 & 0.000000384618980 & 1.047678891593882 & IGD \\
9 & 0.000000000000756 & 0.000000384618980 & 1.047678891593878 & IGD \\
10 & 0.000000000000006 & 0.000000384618980 & 1.047678891593878 & IGD \\
\hline
\end{tabular}
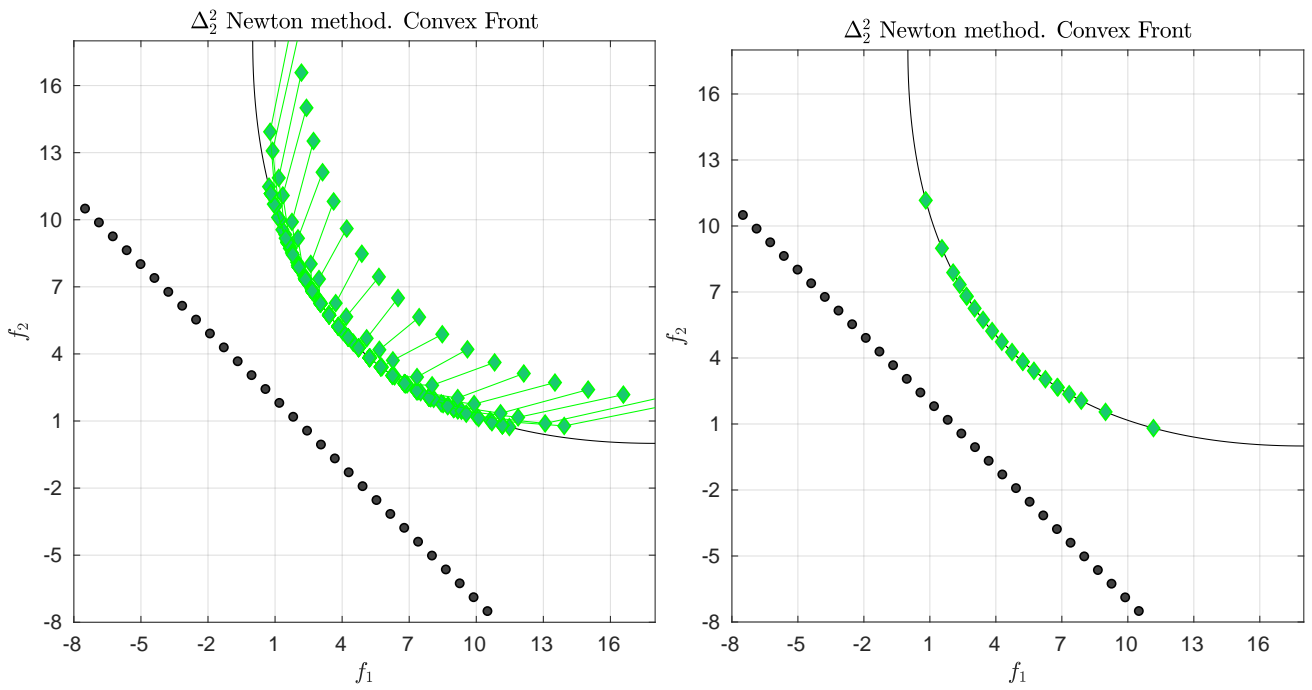

Figure 11. (Left) application of the $\Delta_{2}$-Newton method on BOP (27). (Right) the final archive. 
Table 6. Numerical results of the $\Delta_{2}^{2}$-Newton method on BOP (50), see Figure 12.

\begin{tabular}{|c|c|c|c|c|}
\hline Iter. & $\left\|\nabla \Delta_{2}^{2}\left(A^{i}\right)\right\|$ & $\Delta_{2}^{2}\left(F\left(A^{i}\right), F\left(P_{Q}\right)\right)$ & $\overline{\Delta_{2}^{2}\left(F\left(A^{i}\right), Z\right)}$ & Indicator \\
\hline 0 & & 0.278373584606464 & 0.139932582443422 & GD \\
\hline 1 & 0.056371237267200 & 0.066618294837097 & 0.056728504338161 & GD \\
\hline 2 & 0.057045938719184 & 0.039369161609912 & 0.041433057966044 & GD \\
\hline 3 & 0.037484475625202 & 0.024109339347752 & 0.031977133783097 & IGD \\
\hline 4 & 0.050812222533911 & 0.023513431743364 & 0.033996922698945 & GD \\
\hline 5 & 0.031160653990564 & 0.014395303481718 & 0.024321095970292 & IGD \\
\hline 6 & 0.037264116905168 & 0.016443622791045 & 0.025809212757710 & IGD \\
\hline 7 & 0.018144934519792 & 0.024703492528406 & 0.029965847813991 & IGD \\
\hline 8 & 0.019468781951843 & 0.028619439695385 & 0.030778919683651 & GD \\
\hline 9 & 0.035410330655855 & 0.017309625336888 & 0.019853297293327 & IGD \\
\hline 10 & 0.043091325647137 & 0.021752471483838 & 0.024124052991424 & IGD \\
\hline 11 & 0.008311561314162 & 0.017118271463333 & 0.026490329733338 & GD \\
\hline 12 & 0.029726132461309 & 0.011015521887052 & 0.017647857545199 & IGD \\
\hline 13 & 0.049385612870240 & 0.013547698417436 & 0.021426583957166 & IGD \\
\hline 14 & 0.014525769797194 & 0.020436747509906 & 0.034124722919781 & IGD \\
\hline 15 & 0.030582462613590 & 0.012480668370491 & 0.021750946945761 & IGD \\
\hline 16 & 0.030419387651439 & 0.006090064700256 & 0.023544581385908 & IGD \\
\hline 17 & 0.012758353366778 & 0.000030594174296 & 0.025131789220814 & IGD \\
\hline 18 & 0.009657374280631 & 0.001454430574110 & 0.025813967350062 & IGD \\
\hline 19 & 0.005296333332650 & 0.001374135866037 & 0.026375698139894 & IGD \\
\hline 20 & 0.005548518084090 & 0.002112406054454 & 0.027521017269386 & IGD \\
\hline 21 & 0.005856819919213 & 11375528 & 0.029968509026162 & IGD \\
\hline 22 & 0.012701286040104 & 0.001922080339960 & 0.030132357483057 & IGD \\
\hline 23 & 0.003183819547848 & 0.001504297326063 & 0.030456038027207 & IGD \\
\hline 24 & 0.003253860331803 & 0.002240659587601 & 0.031310708007687 & IGD \\
\hline 25 & 0.003580104890061 & 0.001602870721889 & 0.031465842685442 & IGD \\
\hline 26 & 0.002074689422294 & 0.001367127795787 & 0.031805380040383 & IGD \\
\hline 27 & 0.001414150903872 & 0.000126099902661 & 0.031769100819775 & IGD \\
\hline 28 & 0.001111604812819 & 0.001688362662578 & 0.031742469387990 & IGD \\
\hline 29 & 0.000901680741441 & 0.003036794425943 & 0.031689031421120 & IGD \\
\hline 30 & 0.000257772611116 & 0.003797901156449 & 0.031672123060034 & IGD \\
\hline 31 & 0.000101230696412 & 0.003991409932376 & 0.031663374641811 & IGD \\
\hline 32 & 0.000007716198343 & 0.004008504531546 & 0.031662626381853 & IGD \\
\hline 33 & 0.000000360445308 & 0.004008654325951 & 0.031662593054177 & IGD \\
\hline 34 & 0.000000015185568 & 0.004008654388153 & 0.031662591709428 & IGD \\
\hline 35 & 0.000000000643412 & 0.004008654388154 & 0.031662591654952 & IGD \\
\hline 36 & 0.000000000027461 & 0.004008654388154 & 0.031662591652896 & IGD \\
\hline 37 & 0.000000000001332 & 0.004008654388154 & 0.031662591652858 & IGD \\
\hline 38 & 0.000000000000154 & 0.004008654388154 & 0.031662591652867 & IGD \\
\hline 39 & 000033 & 0.004008654388154 & 2091032009 & GD \\
\hline
\end{tabular}
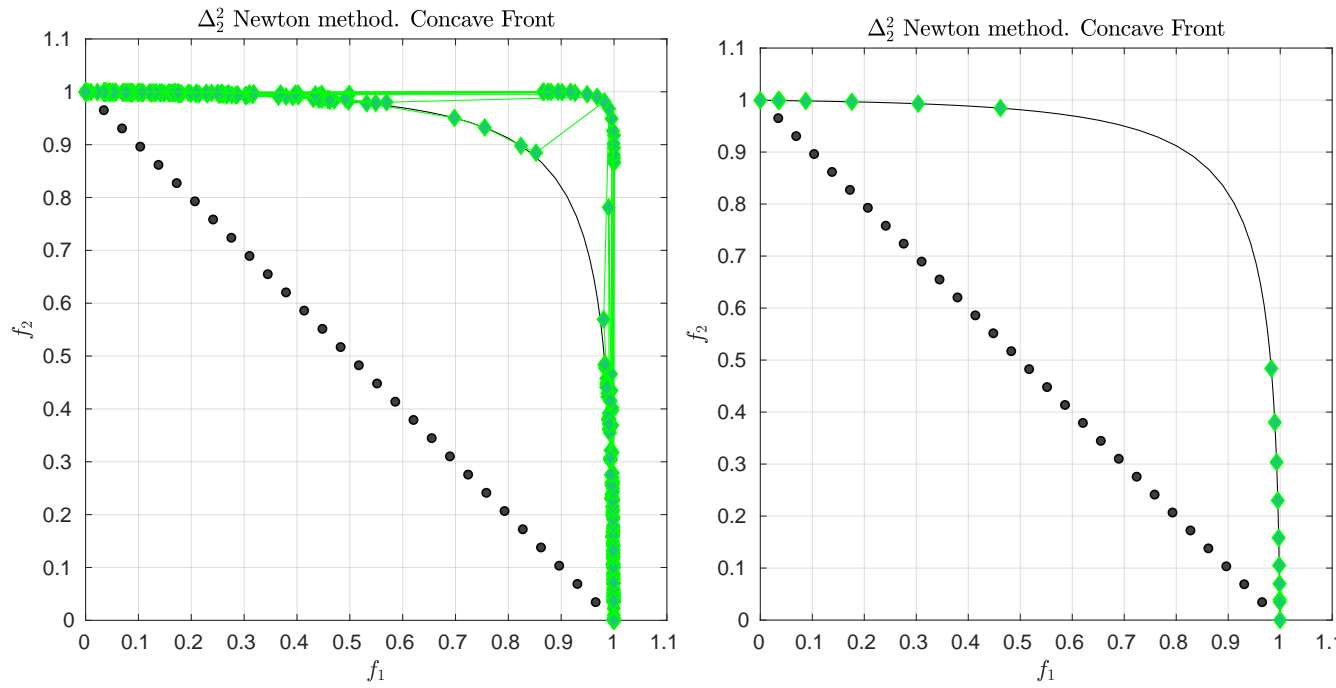

Figure 12. (Left) application of the $\Delta_{2}^{2}$-Newton method on BOP (50). (Right) the final archive. 

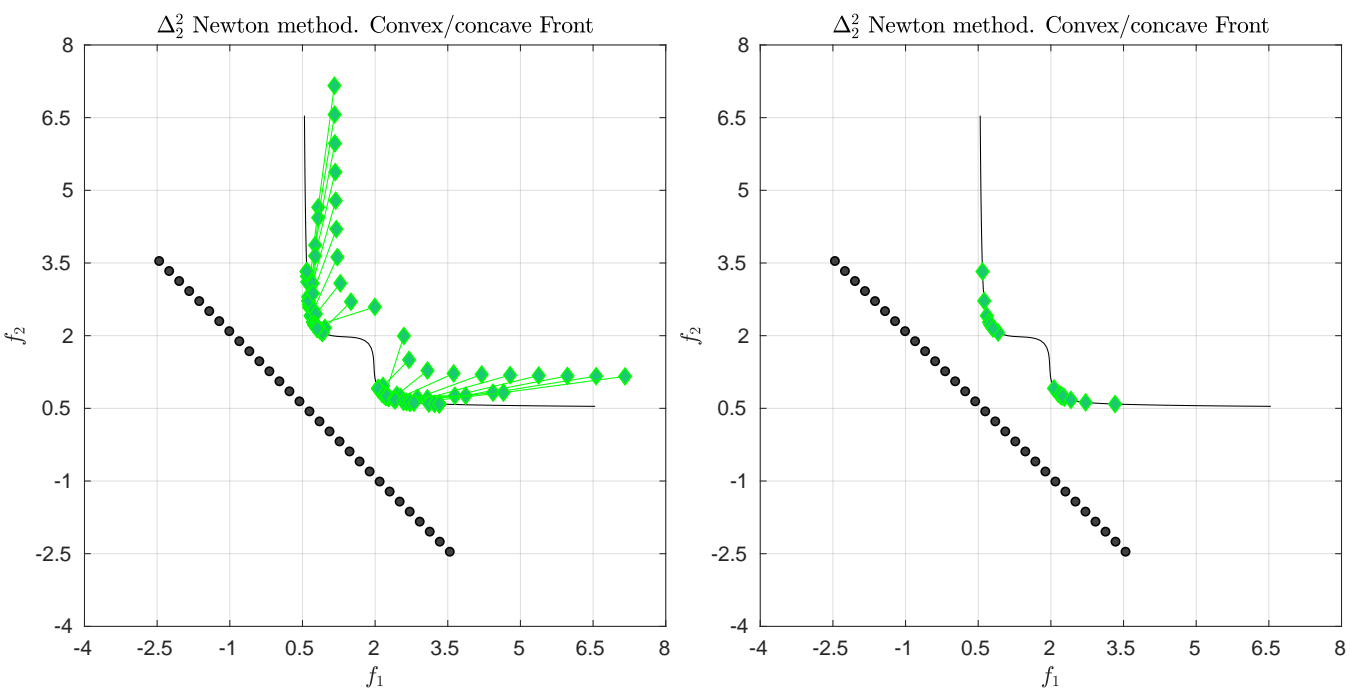

Figure 13. (Left) application of the $\Delta_{2}$-Newton method on BOP (51). (Right) the final archive.

Table 7. Numerical results of the $\Delta_{2}^{2}$-Newton method on BOP (51), see Figure 13.

\begin{tabular}{ccccl}
\hline Iter. & $\left\|\nabla \Delta_{\mathbf{2}}^{\mathbf{2}}\left(A^{\boldsymbol{i}}\right)\right\|$ & $\boldsymbol{\Delta}_{\mathbf{2}}^{\mathbf{2}}\left(\boldsymbol{F}\left(\boldsymbol{A}^{\boldsymbol{i}}\right), \boldsymbol{F}\left(\boldsymbol{P}_{\mathbf{Q}}\right)\right)$ & $\boldsymbol{\Delta}_{\mathbf{2}}^{\mathbf{2}}\left(\boldsymbol{F}\left(A^{\boldsymbol{i}}\right), \boldsymbol{Z}\right)$ & Indicator \\
\hline 0 & - & 0.706541653137130 & 0.794786137846191 & GD \\
1 & 2.052766083969590 & 0.169306894160018 & 0.477606371056880 & GD \\
2 & 1.043651307474400 & 0.001325637478010 & 0.312621943719186 & IGD \\
3 & 0.335281361638164 & 0.000782828935146 & 0.318925114182142 & IGD \\
4 & 0.091979632627269 & 0.000782533520334 & 0.322156690814970 & IGD \\
5 & 0.070361802550103 & 0.000782533238333 & 0.325270306125291 & IGD \\
6 & 0.001111027357469 & 0.000782533238004 & 0.325312843569155 & IGD \\
7 & 0.000000574058899 & 0.000782533238004 & 0.325312858713113 & IGD \\
8 & 0.000000000000187 & 0.000782533238004 & 0.325312858713118 & IGD \\
9 & 0.000000000000000 & 0.000782533238004 & 0.325312858713118 & IGD \\
\hline
\end{tabular}

We next consider an example where we use the unconstrained three-objective problem that is defined by the following map

$$
\begin{aligned}
F: \mathbb{R}^{3} \rightarrow \mathbb{R}^{3} \\
F(x)=\left(\begin{array}{c}
\left(x_{1}+1\right)^{2}+\left(x_{2}+1\right)^{2}+\left(x_{3}+1\right)^{2} \\
\left(x_{1}-1\right)^{2}+\left(x_{2}-1\right)^{2}+\left(x_{3}-1\right)^{2} \\
\left(x_{1}+1\right)^{2}+\left(x_{2}-1\right)^{2}+\left(x_{3}+1\right)^{2}
\end{array}\right) .
\end{aligned}
$$

For this problem, we consider the following scenario: assume there are two decision makers which each have their own preference vector (denote here by $z_{1}=(6.08,-2.08,2.72)^{T}$ and $\left.z_{2}=(0.32,3.68,-3.04)^{T}\right)$. As compromise it could be interesting to consider the line segment that connects $z_{1}$ and $z_{2}$ (denote by $Z$ ) and to compute a set of solutions along the Pareto front that is near (in the Hausdorff sense) to this aspiration set. Figure 14 and Table 8 show the numerical result of the Newton method for an initial set consisting of 7 elements. As anticipated, the final set resembles a curve along the Pareto front with minimal distance to $Z$, and may be used for the decision making process. 

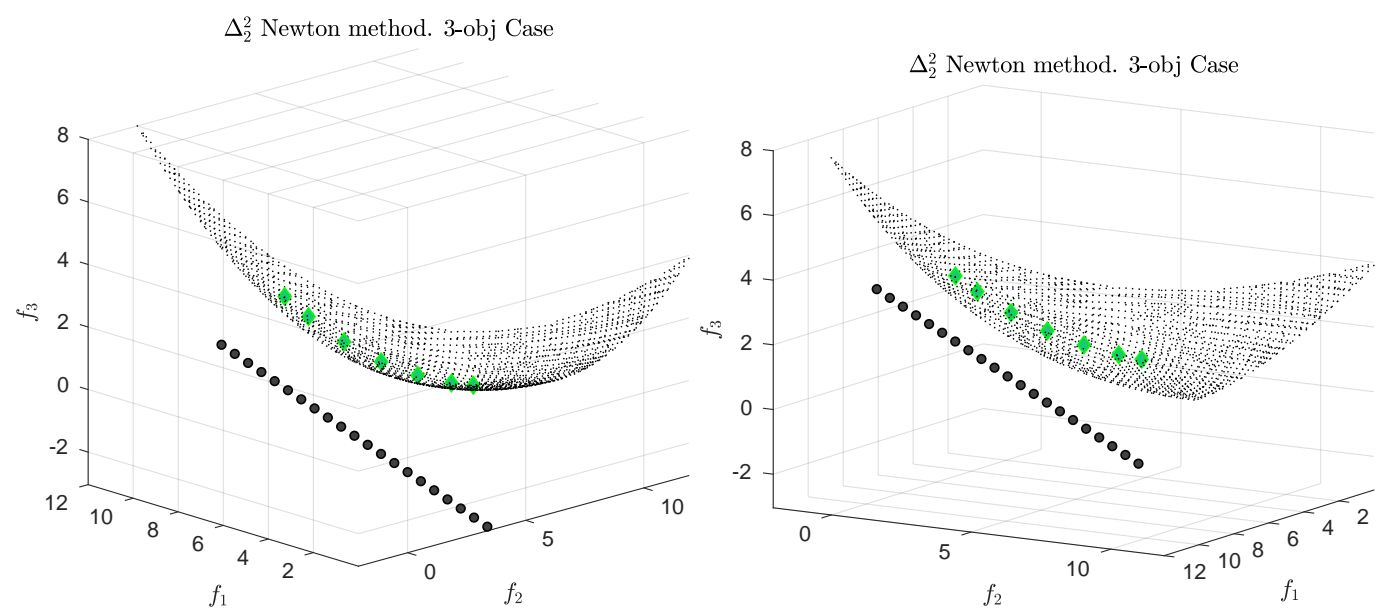

Figure 14. Result of the $\Delta_{2}^{2}$-Newton method for MOP (53), where $Z$ is a line.

This concept can of course be extended to general sets. For instance, one can choose the triangle $Z$ that is defined by the three vertices $z_{1}=(6.08,-2.08,2.72)^{T}, z_{2}=(-2.56,6.56,-0.16)^{T}$ and $z_{3}=(0.20,3.79,-2.92)^{T}$ (e.g., if a third decision maker is involved). Figure 15 and Table 9 show such a numerical result. For sake of better visualization, we only show the edges $Z$ instead of the complete triangle. As it can be seen, the obtained solutions resemble to a certain extent a bended triangle along the Pareto front. From Table 9 it follows that for the final iteration the value $\left\|\nabla \Delta_{2}^{2}\left(A^{6}\right)\right\|$ is already very close to zero which indicates that a local solution has been computed. The solution, however, does not seem to be perfectly shaped which is due to the fact that the problem to locate solutions along the Pareto front with respect to the given reference set is highly multi-modal (the "perfect" shape is associated to the global solution of Problem (5)). In order to obtain better results it is hence imperative to hybridize the set based Newton methods with global multi-objective solvers (as e.g., multi-objective evolutionary algorithms) which is beyond the scope of this work.
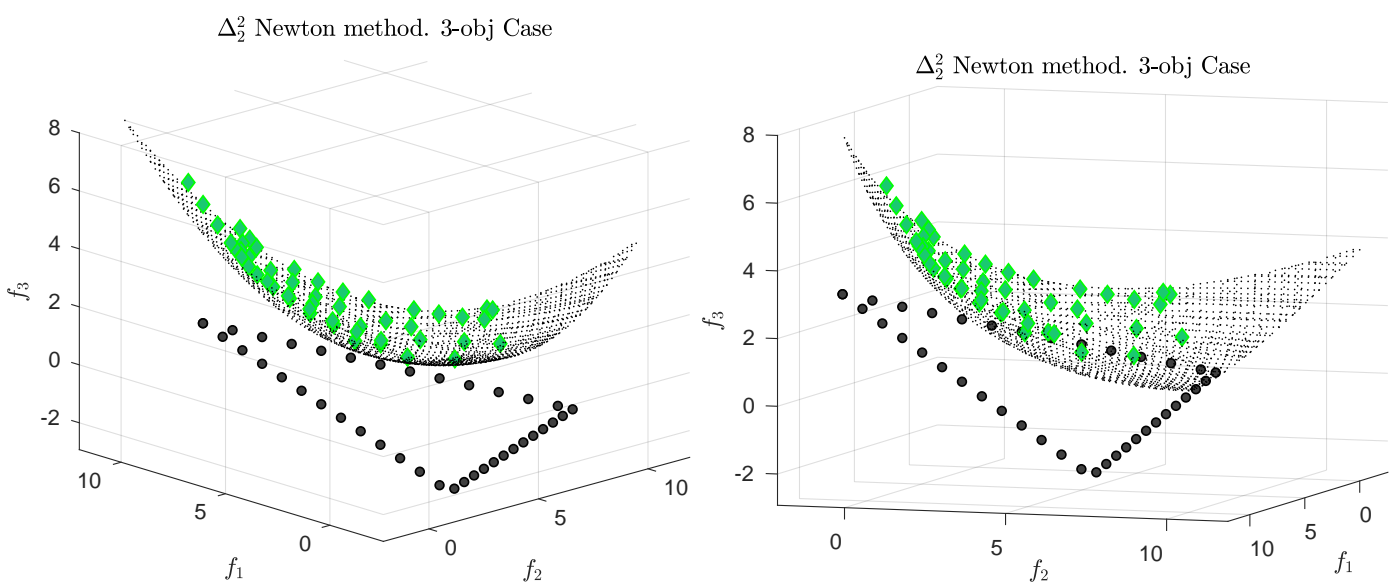

Figure 15. Result of the $\Delta_{2}^{2}$-Newton method for MOP (53), where $Z$ is a triangle (two different views of the same result). 
Table 8. Numerical results of $\Delta_{2}^{2}$-Newton method for MOP (53), see Figure 14 .

\begin{tabular}{cccc}
\hline Iter. & $\left\|\nabla \Delta_{\mathbf{2}}^{\mathbf{2}}\left(A^{\mathbf{i}}\right)\right\|$ & $\boldsymbol{\Delta}_{\mathbf{2}}^{\mathbf{2}}\left(\boldsymbol{F}\left(A^{\boldsymbol{i}}\right), \boldsymbol{F}\left(\boldsymbol{P}_{Q}\right)\right)$ & $\Delta_{\mathbf{2}}^{\mathbf{2}}\left(\boldsymbol{F}\left(A^{\mathbf{i}}\right), \boldsymbol{Z}\right)$ \\
\hline 0 & - & 1.378676581248260 & 5.918796450955248 \\
1 & 7.515915778732357 & 1.244858568391063 & 5.821725966611490 \\
2 & 1.853655193889793 & 1.249239765031169 & 5.811343998211261 \\
3 & 0.145669456936361 & 1.250137056379269 & 5.810973507376134 \\
4 & 0.000671971342724 & 1.250139614137738 & 5.810971792145200 \\
5 & 0.000000033865300 & 1.250139614289546 & 5.810971792043552 \\
6 & 0.000000000000003 & 1.250139614289546 & 5.810971792043552 \\
\hline
\end{tabular}

Table 9. Numerical results of $\Delta_{2}^{2}$-Newton method for MOP (53) when $Z$ is a triangle, see Figure 15.

\begin{tabular}{cccc}
\hline Iter. & $\left\|\nabla \Delta_{\mathbf{2}}^{\mathbf{2}}\left(A^{\boldsymbol{i}}\right)\right\|$ & $\boldsymbol{\Delta}_{\mathbf{2}}^{\mathbf{2}}\left(F\left(A^{\boldsymbol{i}}\right), \boldsymbol{F}\left(\boldsymbol{P}_{\mathbf{Q}}\right)\right)$ & $\boldsymbol{\Delta}_{\mathbf{2}}^{\mathbf{2}}\left(F\left(A^{\boldsymbol{i}}\right), \boldsymbol{Z}\right)$ \\
\hline 0 & 1.000000000000000 & 1.378676581248260 & 5.136345894189382 \\
1 & 9.078968824204878 & 1.190673858342912 & 5.014877171961635 \\
2 & 12.361924917381627 & 1.250986213036476 & 3.359444827553141 \\
3 & 4.188649668005252 & 1.076592897207513 & 3.232994469439562 \\
4 & 0.664877643630374 & 1.053683832191072 & 3.217085086974496 \\
5 & 0.686656379329441 & 1.036451498535567 & 3.213854172896627 \\
6 & 0.005761784413677 & 1.036607563930994 & 3.213850773658971 \\
7 & 0.000001010224622 & 1.036607573211355 & 3.213850772877354 \\
8 & 0.000000000000157 & 1.036607573211355 & 3.213850772877354 \\
9 & 0.000000000000002 & 1.036607573211354 & 3.213850772877354 \\
\hline
\end{tabular}

\subsection{A Bootstrap Method for the Computation of the Pareto Front}

It is known that the proper choice of reference points/sets is a non-trivial task for the use of performance indicators in general when targeting at the entire solution set (e.g., [54-56]). In the following we show some numerical results of a bootstrap method that allows to a certain extent to compute approximations of the entire Pareto fronts of a given MOP without prior knowledge of this set. For this, we adapt the idea proposed in [57] to the context of the set based Newton method: given a performance indicator and a set based SOP of the form (5), one can iteratively approximate the Pareto front of a given problem using the Newton method via the following steps:

1. Compute the minima $x_{i}^{*}$ of the individual objectives $f_{i}, i=1, \ldots, k$. Let $y_{i}^{*}=F\left(x_{i}^{*}\right)$, and let $\tilde{Z}_{0}$ be the convex hull of the $y_{i}^{* \prime}$ s (also called convex hull of individual minima (CHIM) [23]). Let $\delta_{0}>0$ and set

$$
Z_{0}=\tilde{Z}_{0}-\delta_{0}
$$

where $\delta_{0}$ is ideally large enough so that $Z_{0}$ is utopian. Compute a Newton step using $Z_{0}$ leading to the set of candidate solutions $A^{(0)}$.

2. In step $l$ of the iteration, use the set $A^{(l-1)}$ computed in the previous iteration to compute a set $\tilde{Z}_{l}$. This can be done via interpolation of the elements of $A^{(l-1)}$ so that $\tilde{Z}_{l}$ only contains mutually non-dominated elements. As new reference set use

$$
Z_{l}=\tilde{Z}_{l}-\delta_{l}
$$

where $\delta_{l}<\delta_{l-1}$. Compute a Newton step using $Z_{l}$ leading to $A^{(l)}$.

For $k=2$ objectives, the CHIM is simply the line segment that connects $y_{1}^{*}$ and $y_{2}^{*}$. Figures $16-18$ and Tables 10-12 show the results of this bootstrapping method on the MOPs (27), (50), and (51), respectively. Table 13 shows the number of function, Jacobian, and Hessian calls that have been spent for each problem. In our computations, we have chosen $\delta_{0}$ sufficiently large, and $\delta_{l}=\frac{1}{2} \delta_{l-1}$ for the shift parameter. The results show that the entire Pareto fronts can for these examples be approximated via using the Newton method together with the bootstrapping method. While the final approximations can considered to be "good" for the 
problems with the convex and convex/concave Pareto fronts, the final solution for MOP (50) that contains a concave Pareto front is not yet satisfying. Table 11 indicates that the solutions do not even converge toward a local solution (even if more iteration steps are performed). We conjecture that the problem results from the multi-modality of the test function which encourages us further to hybridize the set based Newton method with a global strategy in the future.
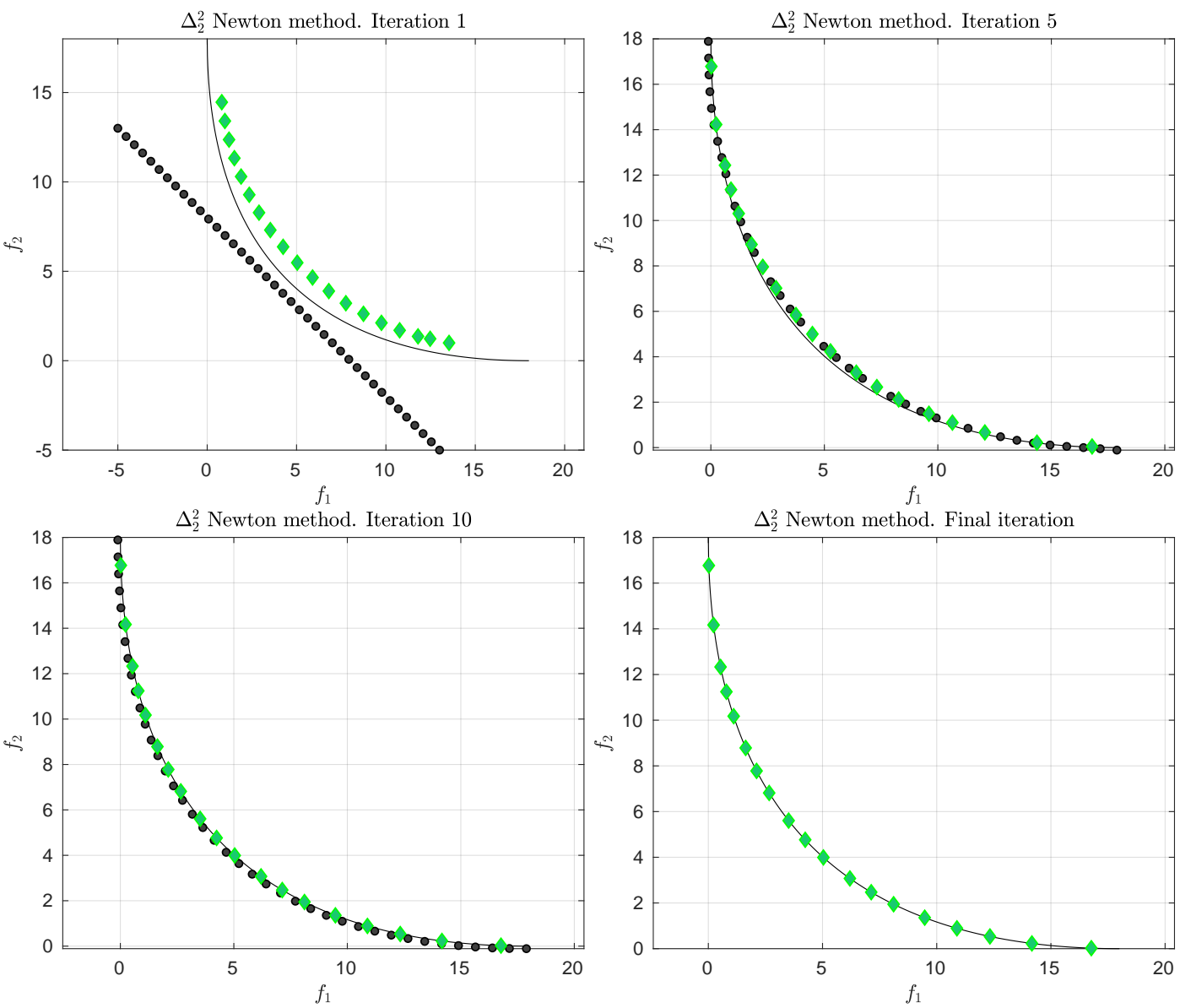

Figure 16. Different iterations of the $\Delta_{2}^{2}$-Newton method to obtain the Pareto front of MOP (27) via the bootstrapping method.

Table 10. Numerical results of the $\Delta_{2}^{2}$-Newton method to obtain the Pareto front of MOP (27) via the bootstrapping method.

\begin{tabular}{ccccc}
\hline Iter. & $\left\|\nabla \Delta_{\mathbf{2}}^{2}\left(A^{i}\right)\right\|$ & $\boldsymbol{\Delta}_{\mathbf{2}}^{\mathbf{2}}\left(\boldsymbol{F}\left(A^{\boldsymbol{i}}\right), \boldsymbol{F}\left(\boldsymbol{P}_{\mathbf{Q}}\right)\right)$ & $\boldsymbol{\Delta}_{\mathbf{2}}^{\mathbf{2}}\left(\boldsymbol{F}\left(\boldsymbol{A}^{\boldsymbol{i}}\right), \boldsymbol{Z}\right)$ & Indicator \\
\hline 0 & - & 2.565838356405802 & 5.454852860388515 & GD \\
1 & 14.958401000284267 & 1.230817708101819 & 1.024752009175881 & IGD \\
2 & 6.553591835159349 & 0.754749784421640 & 0.553744685923295 & IGD \\
3 & 1.930428808338138 & 0.613768117290251 & 0.490492908923838 & IGD \\
4 & 0.937132679630156 & 0.537139549603782 & 0.481517242208329 & IGD \\
5 & 0.540200357139832 & 0.476989307368074 & 0.471988242018486 & IGD \\
6 & 0.394304493982539 & 0.438480476946482 & 0.467546882767516 & IGD \\
7 & 0.153675036875927 & 0.419941366901776 & 0.468192970378975 & IGD \\
8 & 0.059462724070887 & 0.413040100805383 & 0.468716613758660 & IGD \\
9 & 0.039636672484473 & 0.412237873646849 & 0.468845106760589 & IGD \\
10 & 0.016104664984103 & 0.412336536272929 & 0.468844846612736 & IGD \\
11 & 0.001970967225026 & 0.412348016205662 & 0.468845003745375 & IGD \\
12 & 0.000010540592599 & 0.412348100926435 & 0.468845005883349 & IGD \\
13 & 0.000000006447819 & 0.412348100981951 & 0.468845005884675 & IGD \\
14 & 0.000000000000000 & 0.412348100981951 & 0.468845005884675 & IGD \\
\hline
\end{tabular}




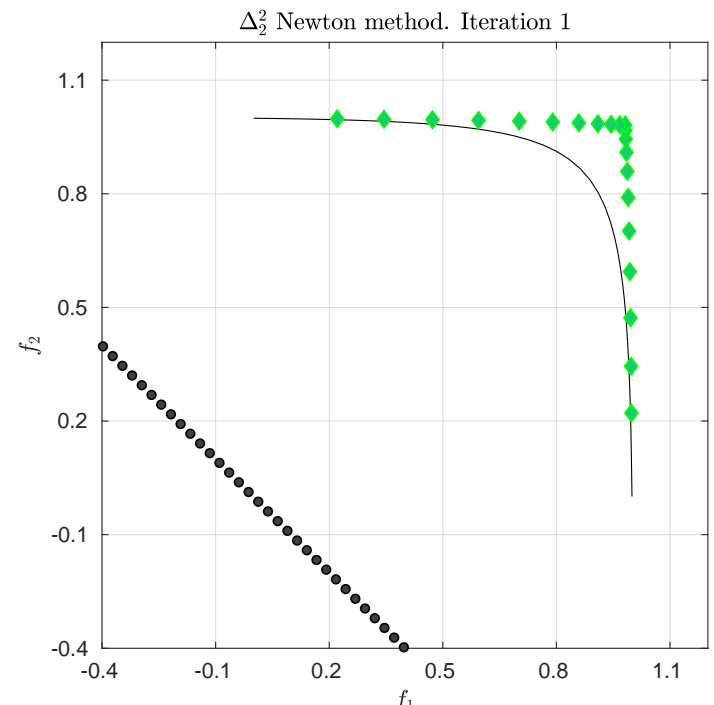

$\Delta_{2}^{2}$ Newton method. Iteration 4

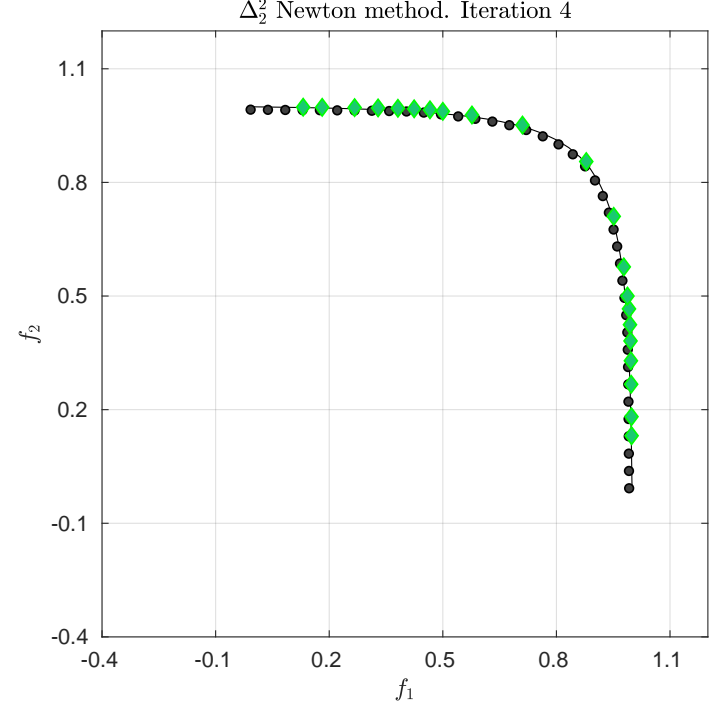

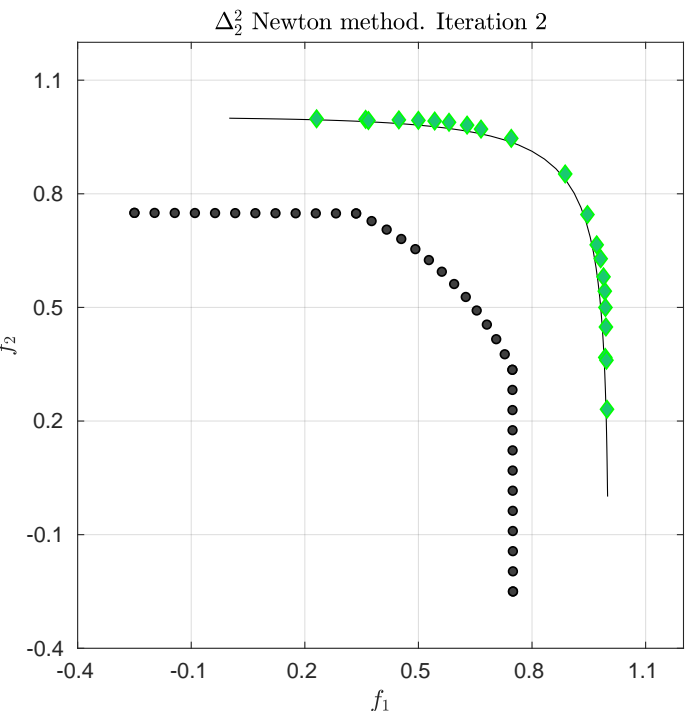

$\Delta_{2}^{2}$ Newton method. Final iteration

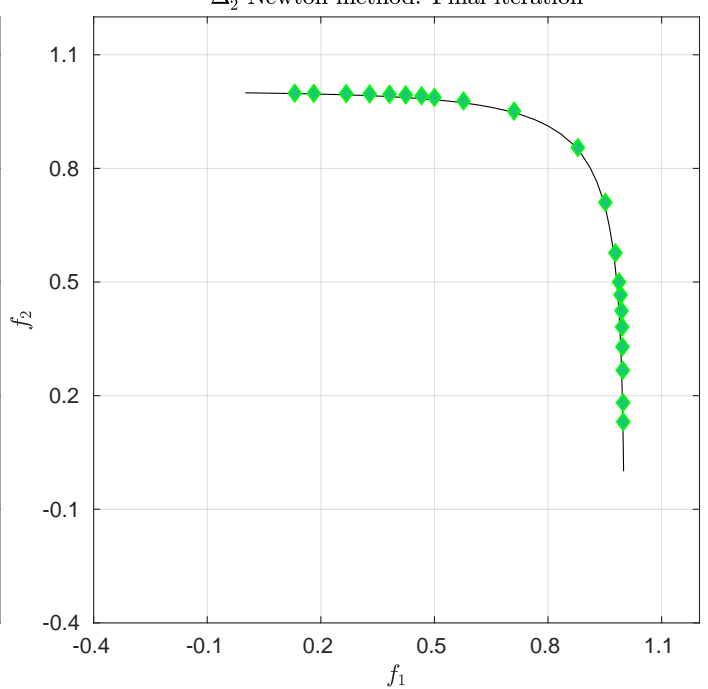

Figure 17. Different iterations of the $\Delta_{2}^{2}$-Newton method to obtain the Pareto front of MOP (50) via the bootstrapping method.

Table 11. Numerical results of the $\Delta_{2}^{2}$-Newton method to obtain the Pareto front of MOP (50) via the bootstrapping method.

\begin{tabular}{ccccc}
\hline Iter. & $\left\|\boldsymbol{\nabla} \boldsymbol{\Delta}_{\mathbf{2}}^{\mathbf{2}}\left(A^{\boldsymbol{i}}\right)\right\|$ & $\boldsymbol{\Delta}_{\mathbf{2}}^{\mathbf{2}}\left(\boldsymbol{F}\left(\boldsymbol{A}^{\boldsymbol{i}}\right), \boldsymbol{F}\left(\boldsymbol{P}_{\mathbf{Q}}\right)\right)$ & $\boldsymbol{\Delta}_{\mathbf{2}}^{\mathbf{2}}\left(\boldsymbol{F}\left(\boldsymbol{A}^{\boldsymbol{i}}\right), \boldsymbol{Z}\right)$ & Indicator \\
\hline 0 & - & 0.455981539616886 & 0.695079920183452 & GD \\
1 & 0.335395116261223 & 0.073901038363798 & 0.755243658407092 & GD \\
2 & 0.091052248527535 & 0.037763808963224 & 0.074896196233522 & IGD \\
3 & 0.014233219389476 & 0.037763808963224 & 0.037618719614753 & IGD \\
4 & 0.012918924846453 & 0.037763808963224 & 0.037607435705178 & IGD \\
5 & 0.012918504651879 & 0.037763808963224 & 0.037607435705178 & IGD \\
\hline
\end{tabular}



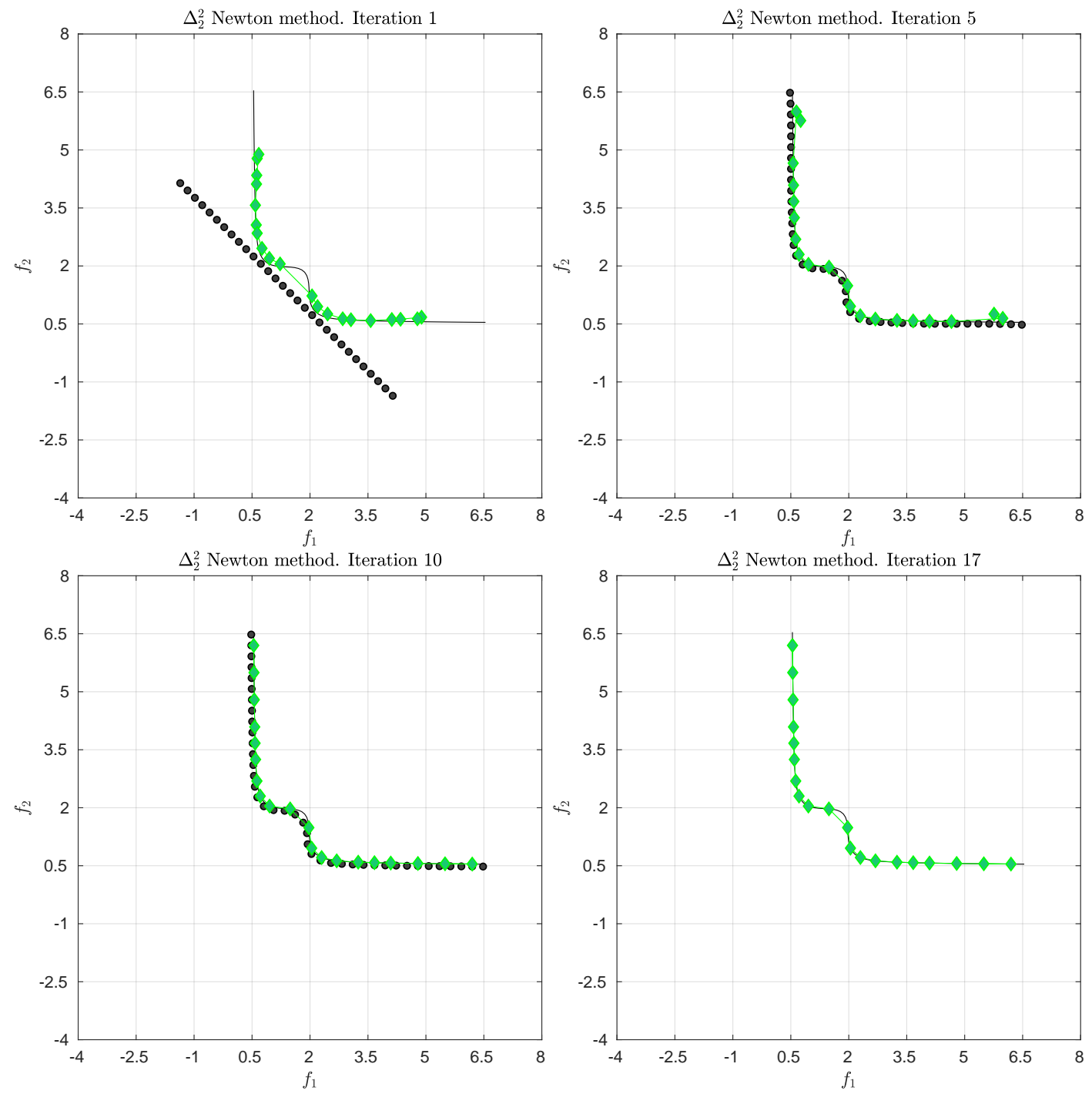

Figure 18. Different iterations of the $\Delta_{2}^{2}$-Newton method to obtain the Pareto front of MOP (51) via the bootstrapping method.

We finally consider a BOP with higher dimensional decision variable space: the bi-objective problem minus DTLZ2 [58] is defined as

$$
\begin{aligned}
& f_{1}(x)=-(1+g(x)) \cos \left(\frac{\pi}{2} x_{1}\right) \\
& f_{2}(x)=-(1+g(x)) \sin \left(\frac{\pi}{2} x_{1}\right) \\
& g(x)=\sum_{i=1}^{n}\left(x_{i}-0.5\right)^{2}
\end{aligned}
$$

where we have chosen $n=20$. Figure 19 shows an initial candidate set as well as the final result of the Newton method together with the bootstrapping. In order to obtain the final result, 8 iteration steps were needed using 1415 function, 700 Jacobian, and 350 Hessian calls. Note that the initial set contains some dominated solutions that do not have an influence on the $I G D_{2}$ value. Hence, also in this case the use of $G D_{2}$ helped to push these solutions toward the Pareto front. 
Table 12. Numerical results of the $\Delta_{2}^{2}$-Newton method to obtain the Pareto front of MOP (51) via the bootstrapping method.

\begin{tabular}{ccccc}
\hline Iter. & $\left\|\nabla \Delta_{2}^{\mathbf{2}}\left(A^{\boldsymbol{i}}\right)\right\|$ & $\boldsymbol{\Delta}_{\mathbf{2}}^{\mathbf{2}}\left(\boldsymbol{F}\left(\boldsymbol{A}^{\mathbf{i}}\right), \boldsymbol{F}\left(\boldsymbol{P}_{\mathbf{Q}}\right)\right)$ & $\boldsymbol{\Delta}_{\mathbf{2}}^{\mathbf{2}}\left(\boldsymbol{F}\left(A^{\boldsymbol{i}}\right), \boldsymbol{Z}\right)$ & Indicator \\
\hline 0 & - & 0.702540625580303 & 2.214433876989687 & GD \\
1 & 1.437150990002929 & 0.389087697290865 & 0.477018278137838 & IGD \\
2 & 0.581565628190262 & 0.342604825739356 & 0.357844471083072 & IGD \\
3 & 0.461927350893728 & 0.164460636656196 & 0.182910255512032 & IGD \\
4 & 0.082455998873464 & 0.158481979725082 & 0.175440371075156 & IGD \\
5 & 0.074464658261760 & 0.191541386471772 & 0.208964468823487 & IGD \\
6 & 0.131398860002112 & 0.153900963788386 & 0.171965063484733 & IGD \\
7 & 0.040291187202238 & 0.152033891842905 & 0.166135305826676 & IGD \\
8 & 0.005689443259245 & 0.151817516262598 & 0.165264799265542 & IGD \\
9 & 0.002103237914802 & 0.151819267034376 & 0.165273277734145 & IGD \\
10 & 0.000466641874904 & 0.151819600203537 & 0.165273337507943 & IGD \\
11 & 0.000013655772453 & 0.151819670338732 & 0.165273320812711 & IGD \\
12 & 0.000000209461574 & 0.151819687437130 & 0.165273316697773 & IGD \\
13 & 0.000000050653504 & 0.151819691559064 & 0.165273315706448 & IGD \\
14 & 0.000000012209033 & 0.151819692552588 & 0.165273315467506 & IGD \\
15 & 0.000000002942920 & 0.151819692792067 & 0.165273315409911 & IGD \\
16 & 0.000000000709377 & 0.151819692849792 & 0.165273315396027 & IGD \\
17 & 0.000000000167849 & 0.151819692849792 & 0.165273315396027 & IGD \\
\hline
\end{tabular}

Table 13. Number of function (\#F), Jacobian $(\# J)$, and Hessian (\#H) calls used by the $\Delta_{2}^{2}$-Newton method using bootstrapping for the three test problems.

\begin{tabular}{cccc}
\hline & MOP (27) & MOP (50) & MOP (51) \\
\hline$\# F$ & 532 & 225 & 698 \\
$\# J$ & 532 & 210 & 680 \\
$\# H$ & 513 & 189 & 660 \\
\hline
\end{tabular}
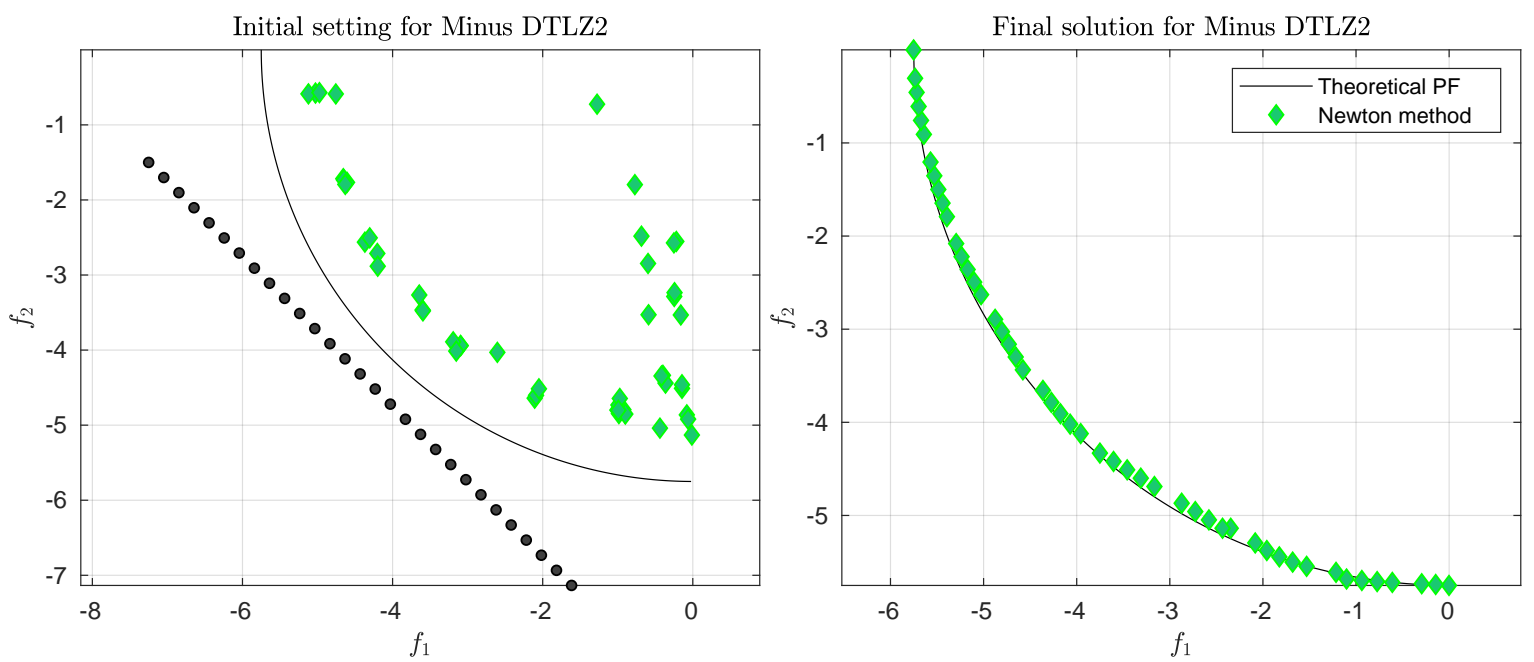

Figure 19. Initial candidate set (left) and numerical result of the $\Delta_{2}$-Newton method on minus DTLZ2 (right).

\section{Conclusions and Future Work}

In this work, we have considered a set based Newton Method for the $\Delta_{p}$ indicator for unconstrained multi-objective optimization problems. Since $\Delta_{p}$ is constructed by GD $D_{p}$ and IGD we have also considered the set based Newton method for these two indicators. To this end, we have first derived the set based gradients and Hessians, and have based on this formulated and analyzed 
the Newton methods. Numerical results on selected test problems have revealed the strengths and weaknesses of the resulting methods. For this, we have mainly considered aspiration set problems (i.e., the problem to minimize the indicator distance of a set to a given utopian reference set) but also shown a bootstapping method that allows to a certain extend to compute finite size approximation of the entire Pareto front without prior knowledge of this set. The results have shown that the method may indeed converge quadratically to the desired set, however, also-and as anticipated-that the Newton method is only applicable locally. It is hence imperative to hybridize the method with a global search strategy such as an evolutionary multi-objective optimization algorithm in order to obtain a fast and reliable algorithm for the treatment of such problems. The latter, however, is beyond the scope of this work and left for future investigations. Another interesting path for future research would be to extend the proposed Newton methods to constrained multi-objective optimization problems.

Author Contributions: Conceptualization and methodology, O.S. and A.L.; formal analysis, J.M.B., A.V., G.R.; validation, L.U. All authors have read and agreed to the published version of the manuscript.

Funding: A.L. acknowledges support from project SIP20201381. O.S. acknowledges support from Conacyt Basic Science project no. 285599 and SEP-Cinvestav project no. 231.

Conflicts of Interest: The authors declare no conflict of interest.

\section{References}

1. Stewart, T.; Bandte, O.; Braun, H.; Chakraborti, N.; Ehrgott, M.; Göbelt, M.; Jin, Y.; Nakayama, H. Real-World Applications of Multiobjective Optimization. In Multiobjective Optimization; Slowinski, R., Ed.; Lecture Notes in Computer Science; Springer: Berlin/Heidelberg, Germany, 2008; Volume 5252, pp. 285-327.

2. Cui, Y.; Geng, Z.; Zhu, Q.; Han, Y. Review: Multi-objective optimization methods and application in energy saving. Energy 2017, 125, 681-704. [CrossRef]

3. Peitz, S.; Dellnitz, M. A Survey of Recent Trends in Multiobjective Optimal Control—Surrogate Models, Feedback Control and Objective Reduction. Math. Comput. Appl. 2018, 23, 30. [CrossRef]

4. Moghadam, M.E.; Falaghi, H.; Farhadi, M. A Novel Method of Optimal Capacitor Placement in the Presence of Harmonics for Power Distribution Network Using NSGA-II Multi-Objective Genetic Optimization Algorithm. Math. Comput. Appl. 2020, 25, 17.

5. Deb, K. Evolutionary multi-objective optimization: Past, present and future. In Proceedings of the 2020 Genetic and Evolutionary Computation Conference Companion, Cancún, Mexico, 8-12 July 2020; pp. 343-372.

6. Hillermeier, C. Nonlinear Multiobjective Optimization: A Generalized Homotopy Approach; Springer Science \& Business Media: Berlin/Heidelberg, Germany, 2001; Volume 135.

7. Zitzler, E.; Thiele, L. Multiobjective evolutionary algorithms: A comparative case study and the strength Pareto approach. IEEE Trans. Evol. Comput. 1999, 3, 257-271. [CrossRef]

8. Van Veldhuizen, D.A. Multiobjective Evolutionary Algorithms: Classifications, Analyses, and New Innovations; Technical Report; Air Force Institute of Technology: Kaduna, Nigeria, 1999.

9. Coello, C.A.C.; Cortés, N.C. Solving Multiobjective Optimization Problems Using an Artificial Immune System. Genet. Program. Evolvable Mach. 2005, 6, 163-190. [CrossRef]

10. Hansen, M.P.; Jaszkiewicz, A. Evaluating the Quality of Approximations of the Non-Dominated Set; IMM Technical Report IMM-REP-1998-7; Institute of Mathematical Modeling, Technical University of Denmark: Kongens Lyngby, Denmark, 1998.

11. Dilettoso, E.; Rizzo, S.A.; Salerno, N. A Weakly Pareto Compliant Quality Indicator. Math. Comput. Appl. 2017, 22, 25. [CrossRef]

12. Schütze, O.; Esquivel, X.; Lara, A.; Coello, C.A.C. Using the averaged Hausdorff distance as a performance measure in evolutionary multi-objective optimization. IEEE Trans. Evol. Comput. 2012, 16, 504-522. [CrossRef]

13. Bogoya, J.M.; Vargas, A.; Schütze, O. The Averaged Hausdorff Distances in Multi-Objective Optimization: A Review. Mathematics 2019, 7, 894. [CrossRef]

14. Deb, K. Multi-Objective Optimization Using Evolutionary Algorithms; John Wiley \& Sons: Chichester, UK, 2001; ISBN 0-471-87339-X. 
15. Deb, K.; Pratap, A.; Agarwal, S.; Meyarivan, T. A fast and elitist multiobjective genetic algorithm: NSGA-II. IEEE Trans. Evol. Comput. 2002, 6, 182-197. [CrossRef]

16. Coello, C.A.C.; Lamont, G.B.; Van Veldhuizen, D.A. Evolutionary Algorithms for Solving Multi-Objective Problems; Springer: Berlin/Heidelberg, Germany, 2007; Volume 5.

17. Beume, N.; Naujoks, B.; Emmerich, M. SMS-EMOA: Multiobjective selection based on dominated hypervolume. Eur. J. Oper. Res. 2007, 181, 1653-1669. [CrossRef]

18. Bringmann, K.; Friedrich, T. Convergence of Hypervolume-Based Archiving Algorithms. IEEE Trans. Evol. Comput. 2014, 18, 643-657. [CrossRef]

19. Powell, M.J.D. On Search Directions for Minimization Algorithms. Math. Program. 1973, 4, $193-201$. [CrossRef]

20. Nocedal, J.; Wright, S. Numerical Optimization; Springer Science \& Business Media: New York, NY, USA, 2006.

21. Bogoya, J.M.; Vargas, A.; Cuate, O.; Schütze, O. A (p, q)-averaged Hausdorff distance for arbitrary measurable sets. Math. Comput. Appl. 2018, 23, 51. [CrossRef]

22. Pascoletti, A.; Serafini, P. Scalarizing vector optimization problems. J. Optim. Theory Appl. 1984, 42, 499-524. [CrossRef]

23. Das, I.; Dennis, J.E. Normal-boundary intersection: A new method for generating the Pareto surface in nonlinear multicriteria optimization problems. SIAM J. Optim. 1998, 8, 631-657. [CrossRef]

24. Ehrgott, M. Multicriteria Optimization; Springer: Berlin/Heidelberg, Germany, 2005.

25. Eichfelder, G. Adaptive Scalarization Methods in Multiobjective Optimization; Springer: Berlin/Heidelberg, Germany, 2008.

26. Miettinen, K. Nonlinear Multi-Objective Optimization; Springer: Berlin/Heidelberg, Germany, 1999 ; Volume 12.

27. Recchioni, M.C. A path following method for box-constrained multiobjective optimization with applications to goal programming problems. Math. Methods Oper. Res. 2003, 58, 69-85. [CrossRef]

28. Schütze, O.; Dell'Aere, A.; Dellnitz, M. On Continuation Methods for the Numerical Treatment of Multi-Objective Optimization Problems. In Practical Approaches to Multi-Objective Optimization; Branke, J., Deb, K., Miettinen, K., Steuer, R.E., Eds.; Number 04461 in Dagstuhl Seminar Proceedings; Internationales Begegnungs- und Forschungszentrum (IBFI): Schloss Dagstuhl, Germany, 2005. Available online: http:/ / drops.dagstuhl.de/opus/volltexte/2005/349 (accessed on 16 October 2020).

29. Pereyra, V.; Saunders, M.; Castillo, J. Equispaced Pareto front construction for constrained bi-objective optimization. Math. Comput. Model. 2013, 57, 2122-2131. [CrossRef]

30. Martin, B.; Goldsztejn, A.; Granvilliers, L.; Jermann, C. Certified Parallelotope Continuation for One-Manifolds. SIAM J. Numer. Anal. 2013, 51, 3373-3401. [CrossRef]

31. Martin, B.; Goldsztejn, A.; Granvilliers, L.; Jermann, C. On continuation methods for non-linear bi-objective optimization: Towards a certified interval-based approach. J. Glob. Optim. 2016, 64, 3-16. [CrossRef]

32. Martín, A.; Schütze, O. Pareto Tracer: A predictor-corrector method for multi-objective optimization problems. Eng. Optim. 2018, 50, 516-536. [CrossRef]

33. Schütze, O.; Cuate, O.; Martín, A.; Peitz, S.; Dellnitz, M. Pareto Explorer: A global/local exploration tool for many-objective optimization problems. Eng. Optim. 2020, 52, 832-855. [CrossRef]

34. Dellnitz, M.; Schütze, O.; Hestermeyer, T. Covering Pareto Sets by Multilevel Subdivision Techniques. J. Optim. Theory Appl. 2005, 124, 113-155. [CrossRef]

35. Jahn, J. Multiobjective search algorithm with subdivision technique. Comput. Optim. Appl. 2006, 35, 161-175. [CrossRef]

36. Schütze, O.; Vasile, M.; Junge, O.; Dellnitz, M.; Izzo, D. Designing optimal low thrust gravity assist trajectories using space pruning and a multi-objective approach. Eng. Optim. 2009, 41, 155-181. [CrossRef]

37. Hsu, C.S. Cell-to-Cell Mapping: A Method of Global Analysis for Nonlinear Systems; Springer Science \& Business Media: Berlin/Heidelberg, Germany, 2013; Volume 64.

38. Hernández, C.; Naranjani, Y.; Sardahi, Y.; Liang, W.; Schütze, O.; Sun, J.Q. Simple Cell Mapping Method for Multi-objective Optimal Feedback Control Design. Int. J. Dyn. Control. 2013, 1, 231-238. [CrossRef]

39. Sun, J.Q.; Xiong, F.R.; Schütze, O.; Hernández, C. Cell Mapping Methods-Algorithmic Approaches and Applications; Springer: Berlin/Heidelberg, Germany, 2019.

40. Juárez-Smith, P.; Trujillo, L.; García-Valdez, M.; Fernández de Vega, F.; Chávez, F. Pool-Based Genetic Programming Using Evospace, Local Search and Bloat Control. Math. Comput. Appl. 2019, 24, 78. [CrossRef] 
41. Sriboonchandr, P.; Kriengkorakot, N.; Kriengkorakot, P. Improved Differential Evolution Algorithm for Flexible Job Shop Scheduling Problems. Math. Comput. Appl. 2019, 24, 80. [CrossRef]

42. Ketsripongsa, U.; Pitakaso, R.; Sethanan, K.; Srivarapongse, T.A. Improved Differential Evolution Algorithm for Crop Planning in the Northeastern Region of Thailand. Math. Comput. Appl. 2018, 23, 40. [CrossRef]

43. Cuate, O.; Schütze, O. Variation Rate to Maintain Diversity in Decision Space within Multi-Objective Evolutionary Algorithms. Math. Comput. Appl. 2019, 24, 3

44. Mohammadi, A.; Omidvar, M.N.; Li, X. Reference point based multi-objective optimization through decomposition. In Proceedings of the 2012 IEEE Congress on Evolutionary Computation, Brisbane, QLD, Australia, 10-15 June 2012; pp. 1-8.

45. Hernandez Mejia, J.A.; Schütze, O.; Cuate, O.; Lara, A.; Deb, K. RDS-NSGA-II: A Memetic Algorithm for Reference Point Based Multi-objective Optimization. Eng. Optim. 2017, 49, 828-845. [CrossRef]

46. Emmerich, M.; Deutz, A. Time complexity and zeros of the hypervolume indicator gradient field. In EVOLVE-A Bridge between Probability, Set Oriented Numerics, and Evolutionary Computation III; Springer: Berlin/Heidelberg, Germany, 2014; pp. 169-193.

47. Zitzler, E.; Thiele, L. Multiobjective optimization using evolutionary algorithms-A comparative case study. In Proceedings of the International Conference on Parallel Problem Solving from Nature, Amsterdam, The Netherlands, 27-30 September 1998; Springer: Berlin/Heidelberg, Germany, 1998; pp. 292-301.

48. Hernández, V.A.S.; Schütze, O.; Wang, H.; Deutz, A.; Emmerich, M. The Set-Based Hypervolume Newton Method for Bi-Objective Optimization. IEEE Trans. Cybern. 2018, 50, 2186-2196. [CrossRef]

49. Fliege, J.; Drummond, L.G.; Svaiter, B.F. Newton's method for multiobjective optimization. SIAM J. Optim. 2009, 20, 602-626. [CrossRef]

50. Baier, R.; Dellnitz, M.; von Molo, M.H.; Sertl, S.; Kevrekidis, I.G. The computation of convex invariant sets via Newton's method. J. Comput. Dyn. 2014, 1, 39-69. [CrossRef]

51. Chong, E.K.; Zak, S.H. An Introduction to Optimization; John Wiley \& Sons: Hoboken, NJ, USA, 2004.

52. Fonseca, C.M.; Fleming, P.J. An overview of evolutionary algorithms in multiobjective optimization. Evol. Comput. 1995, 3, 1-16. [CrossRef]

53. Witting, K. Numerical Algorithms for the Treatment of Parametric Multiobjective Optimization Problems and Applications. Ph.D. Thesis, Deptartment of Mathematics, University of Paderborn, Paderborn, Germany, 2012.

54. Ishibuchi, H.; Imada, R.; Setoguchi, Y.; Nojima, Y. Reference point specification in inverted generational distance for triangular linear Pareto front. IEEE Trans. Evol. Comput. 2018, 22, 961-975. [CrossRef]

55. Ishibuchi, H.; Imada, R.; Setoguchi, Y.; Nojima, Y. How to specify a reference point in hypervolume calculation for fair performance comparison. Evol. Comput. 2018, 26, 411-440. [CrossRef]

56. Li, M.; Yao, X. Quality evaluation of solution sets in multiobjective optimisation: A survey. ACM Comput. Surv. 2019, 52, 1-38. [CrossRef]

57. Schütze, O.; Domínguez-Medina, C.; Cruz-Cortés, N.; de la Fraga, L.G.; Sun, J.Q.; Toscano, G.; Landa, R. A scalar optimization approach for averaged Hausdorff approximations of the Pareto front. Eng. Optim. 2016, 48, 1593-1617. [CrossRef]

58. Ishibuchi, H.; Setoguchi, Y.; Masuda, H.; Nojima, Y. Performance of decomposition-based many-objective algorithms strongly depends on Pareto front shapes. IEEE Trans. Evol. Comput. 2016, 21, 169-190. [CrossRef]

Publisher's Note: MDPI stays neutral with regard to jurisdictional claims in published maps and institutional affiliations.

(C) 2020 by the authors. Licensee MDPI, Basel, Switzerland. This article is an open access article distributed under the terms and conditions of the Creative Commons Attribution (CC BY) license (http:/ / creativecommons.org/licenses/by/4.0/). 